\title{
Sum of Hermitian Matrices with Given Eigenvalues: Inertia, Rank, and Multiple Eigenvalues
}

\author{
Chi-Kwong Li \\ William \& Mary, ckli@wm.edu \\ Yiu-Tung Poon
}

Follow this and additional works at: https://scholarworks.wm.edu/aspubs

\section{Recommended Citation}

Li, C. K., \& Poon, Y. T. (2010). Sum of Hermitian matrices with given eigenvalues: inertia, rank, and multiple eigenvalues. Canadian Journal of Mathematics, 62(1), 109-132.

This Article is brought to you for free and open access by the Arts and Sciences at W\&M ScholarWorks. It has been accepted for inclusion in Arts \& Sciences Articles by an authorized administrator of W\&M ScholarWorks. For more information, please contact scholarworks@wm.edu. 
Canad. J. Math. Vol. 62 (1), 2010 pp. 109-132

doi:10.4153/CJM-2010-007-2

(c) Canadian Mathematical Society 2010

\title{
Sum of Hermitian Matrices with Given Eigenvalues: Inertia, Rank, and Multiple Eigenvalues
}

\author{
Chi-Kwong Li and Yiu-Tung Poon
}

Abstract. Let $A$ and $B$ be $n \times n$ complex Hermitian (or real symmetric) matrices with eigenvalues $a_{1} \geq \cdots \geq a_{n}$ and $b_{1} \geq \cdots \geq b_{n}$. All possible inertia values, ranks, and multiple eigenvalues of $A+B$ are determined. Extension of the results to the sum of $k$ matrices with $k>2$ and connections of the results to other subjects such as algebraic combinatorics are also discussed.

\section{Introduction}

Let $\mathcal{H}_{n}$ be the real linear space of $n \times n$ complex Hermitian (or real symmetric) matrices. For a real vector $\mathbf{a}=\left(a_{1}, \ldots, a_{n}\right)$ with $a_{1} \geq \cdots \geq a_{n}$, let

$$
\mathcal{H}_{n}(\mathbf{a})=\left\{A \in \mathcal{H}_{n}: A \text { has eigenvalues } a_{1}, \ldots, a_{n}\right\} .
$$

Motivated by problems in pure and applied subjects, there has been much research on the relation between the eigenvalues of $A, B \in \mathcal{H}_{n}$ and those of $A+B[3-5,7-9$, $11,12]$. In particular, for given real vectors $\mathbf{a}=\left(a_{1}, \ldots, a_{n}\right), \mathbf{b}=\left(b_{1}, \ldots, b_{n}\right)$, and $\mathbf{c}=\left(c_{1}, \ldots, c_{n}\right)$ with entries arranged in descending order, a necessary and sufficient condition for the existence of $(A, B) \in \mathcal{H}_{n}(\mathbf{a}) \times \mathcal{H}_{n}(\mathbf{b})$ such that $A+B \in \mathcal{H}_{n}(\mathbf{c})$, or equivalently,

$$
\mathcal{H}_{n}(\mathbf{c}) \subseteq \mathcal{H}_{n}(\mathbf{a})+\mathcal{H}_{n}(\mathbf{b})
$$

can be completely described in terms of the equality

$$
\sum_{j=1}^{n}\left(a_{j}+b_{j}-c_{j}\right)=0
$$

and a collection of inequalities in the form

$$
\sum_{r \in R} a_{r}+\sum_{s \in S} b_{s} \geq \sum_{t \in T} c_{t}
$$

Received by the editors May 31, 2007

The first author's research was supported by a USA NSF grant and a HK RCG grant.

AMS subject classification: 15A42, 15A57.

Keywords: complex Hermitian matrices, real symmetric matrices, inertia, rank, multiple eigenvalues. 
for certain $m$ element subsets $R, S, T \subseteq\{1, \ldots, n\}$ with $1 \leq m<n$ determined by the Littlewood-Richardson rules; see [5,7] for details. Using (1.2) and (1.3), we can also deduce the inequalities

$$
\sum_{r \in R^{c}} a_{r}+\sum_{s \in S^{c}} b_{s} \leq \sum_{t \in T^{c}} c_{t}
$$

where $R^{c}$ denotes the complement of $R$ in $\{1,2, \ldots, n\}$. The study has connections to many different areas such as representation theory, algebraic geometry, and algebraic combinatorics.

The set of inequalities in (1.3) grows exponentially with $n$. Therefore, in spite of the existence of a complete description of the eigenvalues of $A+B$ in terms of those of $A$ and $B$ in $\mathcal{H}_{n}$, it is sometimes difficult to answer some basic questions related to the eigenvalues of the matrices $A, B$, and $A+B$. For example, as pointed out by Fulton [7, p.215], given a proper subset $K$ of $\{1,2, \ldots, n\}$ and real numbers $\left\{\gamma_{k}: k \in K\right\}$, it is not easy to use the inequalities in (1.3) to determine if there exists $\mathbf{c}$ with $c_{k}=\gamma_{k}$ for all $k \in K$ such that (1.1) holds. In particular, the inequalities in (1.3) with $T \subseteq K$ together with those in (1.4) with $T^{c} \subseteq K$ are necessary, but not sufficient, for (1.1) in general.

If $K=\{k\}$ is a singleton, then inequalities in (1.3) and (1.4) reduce to Weyl's inequalities [13], implying that $c_{k} \in\left[L_{k}, R_{k}\right]$, where

$$
L_{k}=\max \left\{a_{i}+b_{j}: i+j=n+k\right\} \quad \text { and } \quad R_{k}=\min \left\{a_{i}+b_{j}: i+j=k+1\right\} .
$$

Conversely, one can check that for every $c \in\left[L_{k}, R_{k}\right]$, there exists

$$
(A, B) \in \mathcal{H}_{n}(\mathbf{a}) \times \mathcal{H}_{n}(\mathbf{b})
$$

satisfying $A+B \in \mathcal{H}_{n}(\mathbf{c})$ with $c_{k}=c$. So, in this case, the inequalities in (1.3) with $T \subseteq K$ and $c_{k}=\gamma_{k}$ for $k \in K$ are also sufficient.

In this paper, we show that if $\mu \in\left[L_{k}, L_{k-1}\right) \cap\left(R_{k^{\prime}+1} \cdot R_{k^{\prime}}\right]$, then there exists $(A, B) \in \mathcal{H}_{n}(\mathbf{a}) \times \mathcal{H}_{n}(\mathbf{b})$ such that $C=A+B$ has a vector of eigenvalues $\mathbf{c}$ with

$$
c_{k-1}<\mu=c_{k}=c_{k+1}=\cdots=c_{k^{\prime}}<c_{k^{\prime}+1} .
$$

This will follow from a consequence (Corollary 5.7) of the solution of the following problem.

Problem 1 Suppose $(A, B) \in \mathcal{H}_{n}(\mathbf{a}) \times \mathcal{H}_{n}(\mathbf{b})$. Can a given $\mu \in \mathbf{R}$ be an eigenvalue of $A+B$ with a specific multiplicity? Equivalently, can $A+B-\mu I$ have a specific rank?

We will study the following more difficult problem.

Problem 2 Suppose $(A, B) \in \mathcal{H}_{n}(\mathbf{a}) \times \mathcal{H}_{n}(\mathbf{b})$. Can a given $\mu \in \mathbf{R}$ be an eigenvalue of $A+B$ so that $p$ other eigenvalues are larger than $\mu$, and $q$ other eigenvalues are smaller than $\mu$ ? Equivalently, can $A+B-\mu I$ have inertia $(p, q, n-p-q)$, i.e., $p$ positive eigenvalues, $q$ negative eigenvalues, and $n-p-q$ zero eigenvalues? 
Clearly, one can replace $(A, B)$ by $(A-\mu I, B)$ and replace $\mathbf{a}=\left(a_{1}, \ldots, a_{n}\right)$ by $\left(a_{1}-\mu, \ldots, a_{n}-\mu\right)$ so as to focus on the case for $\mu=0$ in the study.

For two nonnegative integers $p$ and $q$ with $p+q \leq n$, let

$\mathcal{H}_{n}(p, q)=\left\{X \in \mathcal{H}_{n}: X\right.$ has $p$ positive eigenvalues and $q$ negative eigenvalues $\}$.

In Section 2, we determine a necessary and sufficient condition on $(p, q)$ for the existence of $(A, B) \in \mathcal{H}_{n}(\mathbf{a}) \times \mathcal{H}_{n}(\mathbf{b})$ so that $A+B \in \mathcal{H}_{n}(p, q)$. In addition, we give a global description of the set of integer pairs $(p, q)$ satisfying these conditions in Section 3. Moreover, we determine those integer pairs $(p, q)$ for the existence of diagonal matrices $A \in \mathcal{H}_{n}(\mathbf{a})$ and $B \in \mathcal{H}_{n}(\mathbf{b})$ such that $A+B \in \mathcal{H}_{n}(p, q)$ in Section 4. Then the results are used to determine all the possible ranks of matrices of the form $A+B$ with $(A, B) \in \mathcal{H}_{n}(\mathbf{a}) \times \mathcal{H}_{n}(\mathbf{b})$ in Section 5. We also determine the function $f: \mathbf{R} \rightarrow \mathbf{Z}$ such that $f(\mu)$ is the minimum rank of a matrix of the form $A+B-\mu I$ with $(A, B) \in \mathcal{H}_{n}(\mathbf{a}) \times \mathcal{H}_{n}(\mathbf{b})$. Additional remarks and problems are mentioned in Section 6.

Alternatively, one can describe the results as follows. For $(A, B) \in \mathcal{H}_{n}(\mathbf{a}) \times \mathcal{H}_{n}(\mathbf{b})$, we determine the condition on $(p, q)$ such that $U^{*} A U+V^{*} B V \in \mathcal{H}_{n}(p, q)$ for some unitary matrices $U$ and $V$, and use the result to determine all possible ranks and multiplicities of eigenvalues of all matrices of the form $U^{*} A U+V^{*} B V$.

It turns out that it is more convenient to state and prove the results for $A-B$. We will do this in our discussion and focus on the set

$\operatorname{In}(\mathbf{a}, \mathbf{b})=\left\{(p, q) \in \mathbf{Z} \times \mathbf{Z}: \exists(A, B) \in \mathcal{H}_{n}(\mathbf{a}) \times \mathcal{H}_{n}(\mathbf{b})\right.$ such that $\left.A-B \in \mathcal{H}_{n}(p, q)\right\}$

We always assume that $\mathbf{a}=\left(a_{1}, \ldots, a_{n}\right), \mathbf{b}=\left(b_{1}, \ldots, b_{n}\right)$, and $\mathbf{c}=\left(c_{1}, \ldots, c_{n}\right)$ are real vectors with entries arranged in descending order unless specified otherwise.

\section{Characterization of Elements in $\operatorname{In}(\mathbf{a}, \mathbf{b})$}

First, we obtain an easy to check necessary and sufficient condition for $(p, q) \in$ $\operatorname{In}(\mathbf{a}, \mathbf{b})$.

Theorem 2.1 Let $\mathbf{a}=\left(a_{1}, \ldots, a_{n}\right)$ and $\mathbf{b}=\left(b_{1}, \ldots, b_{n}\right)$ be real vectors with entries arranged in descending order. Suppose $p$ and $q$ are nonnegative integers satisfying $p+q \leq n$. Then $(p, q) \in \operatorname{In}(\mathbf{a}, \mathbf{b})$ if and only if

(i) $\left(a_{1}, \ldots, a_{n-q}\right)-\left(b_{q+1}, \ldots, b_{n}\right)$ is a nonnegative vector with at least $p$ positive entries, and

(ii) $\left(b_{1}, \ldots, b_{n-p}\right)-\left(a_{p+1}, \ldots, a_{n}\right)$ is a nonnegative vector with at least $q$ positive entries.

Moreover, if (ii) and (iii) hold, then there exist block diagonal matrices

$$
A=A_{1} \oplus \cdots \oplus A_{p+q} \in \mathcal{H}_{n}(\mathbf{a}) \quad \text { and } \quad B=B_{1} \oplus \cdots \oplus B_{p+q} \in \mathcal{H}_{n}(\mathbf{b})
$$

with the same block sizes such that $A_{j}-B_{j}$ is rank one positive definite for $j=1, \ldots, p$ and $A_{j}-B_{j}$ is rank one negative semi-definite for $j=p+1, \ldots, p+q$. 
Remark 2.2 For fixed $p, q \geq 0$ with $p+q \leq n$, let $K=\{p+1, \ldots, n-q\}$. The necessity of condition (ii) and (ii) in Theorem 2.1 can be deduced from the inequalities in (1.3) with $T \subseteq K$ and $c_{k}=0$ for $k \in K$. We will give a direct proof of this result for completeness.

It is convenient to use the following notation in our discussion. Suppose $\mathbf{u}=$ $\left(u_{1}, \ldots, u_{m}\right)$ and $\mathbf{v}=\left(v_{1}, \ldots, v_{m}\right)$ are real vectors with entries arranged in descending order. Write $\mathbf{u} \geq_{k} \mathbf{v}$ (respectively, $\mathbf{u}>_{k} \mathbf{v}$ ) if $\mathbf{u}-\mathbf{v}$ is a nonnegative vector with at least (respectively, exactly) $k$ positive entries. We will use $\mathbf{u} \geq \mathbf{v}$ and $\mathbf{u}>\mathbf{v}$ for $\mathbf{u} \geq_{0} \mathbf{v}$ and $\mathbf{u}>_{0} \mathbf{v}$, respectively. For $\mathbf{a}=\left(a_{1}, \ldots, a_{n}\right)$ and $1 \leq m \leq n$, let $\mathbf{a}^{m}=\left(a_{1}, \ldots, a_{m}\right)$ and $\mathbf{a}_{m}=\left(a_{n-m+1}, \ldots, a_{n}\right)$. One can use these notations to restate conditions (i) and (ii) in Theorem 2.1 as

$$
\mathbf{a}^{n-q} \geq_{p} \mathbf{b}_{n-q} \quad \text { and } \quad \mathbf{b}^{n-p} \geq_{q} \mathbf{a}_{n-p}
$$

The following lemmas are needed to prove Theorem 2.1 The first one was proved in [6].

Lemma 2.3 Let $\tilde{\mathbf{a}}=\left(\tilde{a}_{1}, \ldots, \tilde{a}_{m}\right)$ and $\mathbf{a}=\left(a_{1}, \ldots, a_{n}\right)$ be real vectors with entries arranged in descending order, where $1 \leq m<n$. Then there is $(A, \tilde{A}) \in \mathcal{H}_{n}(\mathbf{a}) \times \mathcal{H}_{m}(\tilde{\mathbf{a}})$ with $\tilde{A}$ as the leading principal submatrix of $A$ if and only if $a_{j} \geq \tilde{a}_{j} \geq a_{n-m+j}$ for $j=1, \ldots, m$.

Lemma 2.4 Let $(A, B) \in \mathcal{H}_{n}(\mathbf{a}) \times \mathcal{H}_{n}(\mathbf{b})$. If $A-B$ is a rank $k$ positive semi-definite matrix, then $\mathbf{a} \geq_{k} \mathbf{b}$.

Proof Applying a suitable unitary similarity to $A-B$, we may assume that $A-B=$ $\operatorname{diag}\left(d_{1}, \ldots, d_{k}, 0, \ldots, 0\right)$ with $d_{1} \geq \cdots \geq d_{k}>0$. Let $C=B+d_{k} I_{k} \oplus 0_{n-k}$ have eigenvalues $c_{1} \geq \cdots \geq c_{n}$. Then using the positive semi-definite ordering, we have

$$
A \geq C \quad \text { and } \quad B+d_{k} I \geq C \geq B
$$

By Weyl's inequalities (see [13]), we have

$$
a_{j} \geq c_{j} \quad \text { and } \quad b_{j}+d_{k} \geq c_{j} \geq b_{j}, \quad j=1, \ldots, n .
$$

Since

$$
k d_{k}=\operatorname{tr}(C-B)=\sum_{j=1}^{n}\left(c_{j}-b_{j}\right)
$$

and each of the summands on the right side is bounded by $d_{k}$, we see that at least $k$ of the summands are positive. It follows that there are at least $k$ indices $j$ such that $a_{j}>b_{j}$.

Lemma 2.5 Let $\mathbf{a}$ and $\mathbf{b}$ be real vectors. Suppose $\left\{a_{1}, a_{2}, \ldots, a_{n}\right\}$ and $\left\{b_{1}, b_{2}, \ldots, b_{n}\right\}$ can be partitioned as

$$
\left\{a_{1}, a_{2}, \ldots, a_{n}\right\}=\bigcup_{j=1}^{r}\left\{a_{j, 1}, \ldots, a_{j, n_{j}}\right\} \quad \text { and } \quad\left\{b_{1}, b_{2}, \ldots, b_{n}\right\}=\bigcup_{j=1}^{r}\left\{b_{j, 1}, \ldots b_{j, n_{j}}\right\}
$$


such that for each $1 \leq j \leq r$,

$$
a_{j, 1} \geq b_{j, 1} \geq a_{j, 2} \geq b_{j, 2} \geq \cdots \geq a_{j, n_{j}} \geq b_{j, n_{j}}
$$

with $a_{j, i}>b_{j, i}$ for at least $k_{j} i$ 's and $\sum_{j=1}^{r} k_{j} \geq m$. Then there exist block diagonal matrices $A=A_{1} \oplus \cdots \oplus A_{m} \in \mathcal{H}_{n}(\mathbf{a})$ and $B=B_{1} \oplus \cdots \oplus B_{m} \in \mathcal{H}_{n}(\mathbf{b})$ with the same block sizes such that $A_{j}-B_{j}$ is rank one positive definite for $j=1, \ldots, m$. Consequently, $(m, 0) \in \operatorname{In}(\mathbf{a}, \mathbf{b})$.

Proof Suppose $r=1$. We prove the statement by induction on $m$. When $m=1$ we have

$$
a_{1} \geq b_{1} \geq a_{2} \geq b_{2} \geq \cdots \geq a_{n} \geq b_{n}
$$

and $a_{i}>b_{i}$ for at least one $i$. If $b_{n} \geq 0$, then by Lemma 2.4 there is $\tilde{A} \in \mathcal{H}_{n+1}$ with eigenvalues $a_{1} \geq \cdots \geq a_{n} \geq a_{n+1}=0$ such that the leading $n \times n$ submatrix has eigenvalues $b_{1} \geq \cdots \geq b_{n}$. Since $\tilde{A}$ is singular, there is $R \in M_{n}$ and $v \in \mathbf{C}^{n}$ such that $\tilde{A}=[R \mid v]^{*}[R \mid v]$. Note that $B=R R^{*}$ and $R^{*} R$ have the same eigenvalues $b_{1} \geq \cdots \geq b_{n}$, and the eigenvalues of $A=[R \mid v][R \mid v]^{*}=R R^{*}+v v^{*}$ are the same as the $n$ largest of $\tilde{A}$ and equal to $a_{1} \geq \cdots \geq a_{n}$. Thus, there exists unitary $A-B=v v^{*}$ is rank one positive semi-definite. If $b_{n}<0$, apply the argument to $A-b_{n} I$ and $B-b_{n} I$ to get the conclusion.

Suppose the result holds for some $m \geq 1$, and (2.5) holds with $a_{i}>b_{i}$ for at least $m+1$ i's. Let $s=\min \left\{i: a_{i}>b_{i}\right\}$. Then by induction assumption, there exist $A_{1}, B_{1} \in \mathcal{H}_{s}$ with eigenvalues $a_{1}, \ldots, a_{s}$ and $b_{1}, \ldots, b_{s}$ and block diagonal matrices $A_{2} \oplus \cdots \oplus A_{m+1}$ and $B_{2} \oplus \cdots \oplus B_{m+1} \in \mathcal{H}_{n-s}$ with eigenvalues $a_{s+1}, \ldots, a_{n}$ and $b_{s+1}, \ldots, b_{n}$ such that $A_{j}-B_{j}$ is rank one positive definite for $j=1, \ldots, m+1$. Thus, $A=A_{1} \oplus A_{2} \oplus \cdots \oplus A_{m+1} \in \mathcal{H}_{n}(\mathbf{a})$ and $B=B_{1} \oplus B_{2} \oplus \cdots \oplus B_{m+1} \in \mathcal{H}_{n}(\mathbf{b})$ satisfy the requirement.

Now, suppose $r>1$. Choose non-negative numbers $\ell_{j}$ with $\min \left\{1, k_{j}\right\} \leq \ell_{j} \leq k_{j}$ for $1 \leq j \leq m$ such that $\ell_{1}+\cdots+\ell_{m}=m$. By the result when $r=1$, there exist block diagonal matrices $A_{j}$ and $B_{j} \in \mathcal{H}_{n_{j}}$ with eigenvalues $a_{j, 1}, \ldots, a_{j, n_{j}}$ and $b_{j, 1}, \ldots b_{j, n_{j}}$ such that $A_{j}-B_{j}$ is positive semi-definite with rank $\ell_{j}$. Thus, for $A=A_{1} \oplus \cdots \oplus A_{m}$ and $B=B_{1} \oplus \cdots \oplus B_{m}, A-B$ is positive semi-definite with rank $m$.

We are now ready to present the proof.

Proof of Theorem 2.1 Suppose $(A, B) \in \mathcal{H}_{n}(\mathbf{a}) \times \mathcal{H}_{n}(\mathbf{b})$ satisfies $A-B \in \mathcal{H}_{n}(p, q)$. Applying a unitary similarity to $A-B$, we may assume that $A-B=\operatorname{diag}\left(c_{1}, \ldots, c_{n}\right)$ such that $c_{1} \geq \cdots \geq c_{p}>0=c_{p+1}=\cdots=c_{n-q}=0>c_{n-q+1} \geq \cdots \geq c_{n}$. Let

$$
A=\left(\begin{array}{ll}
A_{11} & A_{12} \\
A_{21} & A_{22}
\end{array}\right) \quad \text { and } B=\left(\begin{array}{ll}
B_{11} & B_{12} \\
B_{21} & B_{22}
\end{array}\right)
$$

with $A_{11}, B_{11} \in \mathcal{H}_{n-q}$. Then $A_{11}-B_{11}$ is positive semi-definite with $p$ positive eigenvalues. Suppose $A_{11}$ and $B_{11}$ have eigenvalues $\alpha_{1} \geq \cdots \geq \alpha_{n-q}$ and $\beta_{1} \geq \cdots \geq \beta_{n-q}$, respectively. By Lemmas 2.3 and 2.4 , we have

$$
\left(a_{1}, \ldots, a_{n-q}\right) \geq\left(\alpha_{1}, \ldots, \alpha_{n-q}\right) \geq_{p}\left(\beta_{1}, \ldots, \beta_{n-q}\right) \geq\left(b_{q+1}, \ldots, b_{n}\right) .
$$


This proves (ii). Similarly, we can prove condition (ii).

To prove the converse, given real vectors $\mathbf{a}$ and $\mathbf{b}$, we first show that for every $n$, the result holds if $p q=0$ or $p+q=n$. If $(p, q)=(0,0)$, then we have $\mathbf{a}=\mathbf{b}$ and the result follows.

Suppose $p>0$ and $q=0$. Let $n=r p+s$, with $r \geq 0$ and $1 \leq s \leq p$ (not $0 \leq s<p$ as given by the Euclidean algorithm). Then (i) and (ii) imply that

$$
\begin{array}{ll}
a_{i} \geq b_{i} \geq a_{p+i} \geq \cdots \geq a_{r p+i} \geq b_{r p+i} & \text { for } 1 \leq i \leq s, \\
a_{j} \geq b_{j} \geq a_{p+j} \geq \cdots \geq a_{(r-1) p+j} \geq b_{(r-1) p+j} & \text { for } s+1 \leq j \leq p,
\end{array}
$$

with $a_{i}>b_{i}$ for at least $p i$ 's. Therefore the result follows from Lemma 2.5

Similarly, the result holds for $p=0$ and $q>0$. Hence, the result holds if $p q=0$.

For $p+q=n$, let $A=\operatorname{diag}\left(a_{1}, \ldots, a_{n}\right)$ and $B=\operatorname{diag}\left(b_{q+1}, \ldots, b_{n}, b_{1}, \ldots, b_{n-p}\right)$. Then it follows from (i) and (ii) that $A-B \in \mathcal{H}_{n}(p, q)$.

We complete the proof of the converse by induction on $n$. The result is clear for $n \leq 2$

Assume that the result is valid for all real vectors of lengths less than $n$. Suppose $(p, q) \geq(1,1), p+q<n$, and the inequalities in (i) and (ii) hold. Then we have

$$
a_{i} \geq b_{q+i} \text { for } 1 \leq i \leq n-q
$$

and

$$
b_{i} \geq a_{p+i} \text { for } 1 \leq i \leq n-p
$$

with at least $p$ strict inequalities holding in (2.6) and at least $q$ strict inequalities holding in 2.7.

If $a_{i}=b_{q+i}$ for some $1 \leq i \leq n-q$, then letting $\mathbf{a}^{\prime}=\left(a_{1}, \ldots, a_{i-1}, a_{i+1}, \ldots, a_{n}\right)$ and $\mathbf{b}^{\prime}=\left(b_{1}, \ldots, b_{q+i-1}, b_{q+i+1}, \ldots, b_{n}\right)$, we have

$$
\begin{gathered}
1 \leq j<i \Rightarrow a_{j}^{\prime}=a_{j} \geq b_{q+j}=b_{q+j}^{\prime}, \\
i \leq j \leq n-1-q \Rightarrow a_{j}^{\prime}=a_{j+1} \geq b_{q+j+1}=b_{q+j}^{\prime}, \\
1 \leq j<i-p \Rightarrow b_{j}^{\prime}=b_{j} \geq a_{p+j}=a_{p+j}^{\prime}, \\
i-p \leq j<i+q \Rightarrow b_{j}^{\prime}=b_{j} \geq a_{p+j} \geq a_{p+j+1}=a_{p+j}^{\prime} \\
i+q \leq j \leq n-1-p \Rightarrow b_{j}^{\prime}=b_{j+1} \geq a_{p+j+1}=a_{p+j}^{\prime},
\end{gathered}
$$

with at least $p$ strict inequalities holding in (2.8) and at least $q$ strict inequalities holding in (2.9). By the induction hypothesis, there exist $A^{\prime}, B^{\prime} \in \mathcal{H}_{n-1}$ with eigenvalues $a_{1}, \ldots, a_{i-1}, a_{i+1}, \ldots, a_{n}$ and $b_{1}, \ldots, b_{q+i-1}, b_{q+i+1}, \ldots, b_{n}$ such that $A^{\prime}-B^{\prime} \in \mathcal{H}_{n-1}(p, q)$. Hence, $\left[a_{i}\right] \oplus A^{\prime}-\left[b_{q+i}\right] \oplus B^{\prime} \in \mathcal{H}_{n}(p, q)$.

Similarly, the result holds if $b_{i}=a_{p+i}$ for some $1 \leq i \leq n-p$.

So, we may assume that all inequalities are strict in (2.6) and 2.7). By symmetry, we may assume that $q \leq p$. Since $n>p+q$, let $n=r(p+q)+s$, where $r>0$ and 
$1 \leq s \leq p+q$. We will arrange $a_{1}, \ldots, a_{n}$ and $b_{1}, \ldots, b_{n}$ in $p+q$ chains of inequalities so that Lemma 2.5 can be applied. To this end, define $m=\min \{s, q, p+q-s\}$,

$i_{1}=\max \{1, s-q+1\}, i_{2}=\min \{s, p\}, j_{1}=\max \{1, s-p+1\}, j_{2}=\min \{s, q\}$.

We have

\begin{tabular}{|l|c|c|c|}
\hline & $1 \leq s \leq q$ & $q<s \leq p$ & $p<s \leq p+q$ \\
\hline$i_{1}=\max \{1, s-q+1\}$ & 1 & $s-q+1$ & $s-q+1$ \\
\hline$i_{2}=\min \{s, p\}$ & $s$ & $s$ & $p$ \\
\hline$j_{1}=\max \{1, s-p+1\}$ & 1 & 1 & $s-p+1$ \\
\hline$j_{2}=\min \{s, q\}$ & $s$ & $q$ & $q$ \\
\hline$m=\min \{s, q, p+q-s\}$ & $s$ & $q$ & $p+q-s$ \\
\hline
\end{tabular}

Then $i_{2}-i_{1}=j_{2}-j_{1}=m-1$. By conditions (i) and (ii), we can list all the entries of $\mathbf{a}$ and $\mathbf{b}$ in the following $p+q$ chains of interlacing inequalities:

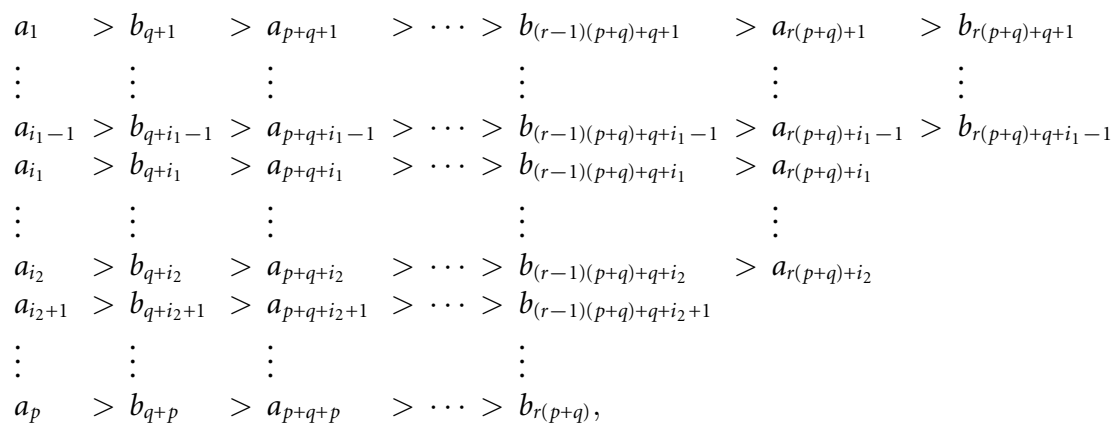

and

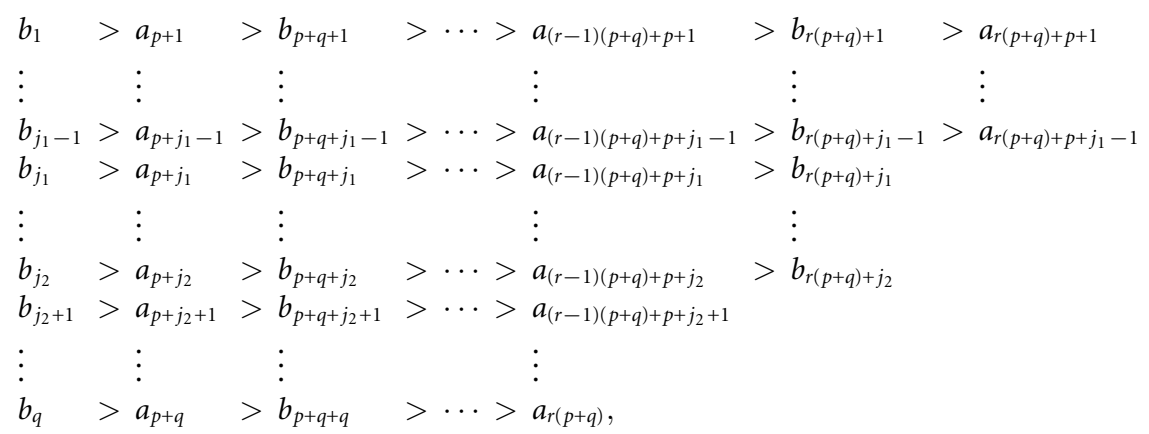

where $a_{i}$ and $b_{i}$ would not appear if $i<0$ or $i>n$.

In fact, it is easy to construct the $p$ chains of inequalities in the first list and $q$ chains of inequalities in the second list as follows. Put the first $p$ entries of a vertically in the first column of the first list, the next $q$ entries of a vertically in the second column of the second list, then the next $p$ entries of $\mathbf{a}$ in the third column of first list, and so 
forth. Similarly, put the first $q$ entries of $\mathbf{b}$ in the first column of the second list, the next $p$ entries of $\mathbf{b}$ in the second column of the first list, then the next $q$ entries of $\mathbf{b}$ in the third column of the second list, and so forth.

For the application of Lemma 2.5, the chains of inequalities with starting terms $a_{i}$ for $i_{1} \leq i \leq i_{2}$ are not acceptable because the first and last terms are entries of a. Similarly, the chains of inequalities with starting terms $b_{j}$ for $j_{1} \leq j \leq j_{2}$ are not acceptable. Since $i_{2}-i_{1}=j_{2}-j_{1}$, we can amend the situations as follows. For $i_{1} \leq i \leq i_{2}$, let $i^{\prime}=j_{1}+i-i_{1}$. Then $j_{1} \leq i^{\prime} \leq j_{2}$ and we can replace the pair of interlacing inequalities

$$
\begin{aligned}
& a_{i}>b_{q+i}>a_{p+q+i}>\cdots>b_{(r-1)(p+q)+q+i}>a_{r(p+q)+i} \\
& b_{i^{\prime}}>a_{p+i^{\prime}}>b_{p+q+i^{\prime}}>\cdots>a_{(r-1)(p+q)+p+i^{\prime}}>b_{r(p+q)+i^{\prime}}
\end{aligned}
$$

by one of the following pairs:

$$
\begin{aligned}
& \quad \begin{array}{l}
a_{i}>b_{q+i}>a_{p+q+i}>\cdots>b_{(r-1)(p+q)+q+i}>a_{r(p+q)+i}>b_{r(p+q)+i^{\prime}}, \\
b_{i^{\prime}}>a_{p+i^{\prime}}>b_{p+q+i^{\prime}}>\cdots>a_{(r-1)(p+q)+p+i^{\prime}}
\end{array} \\
& \text { if } a_{r(p+q)+i}>b_{r(p+q)+i^{\prime}} \text { or } \\
& \qquad \begin{array}{l}
a_{i}>b_{q+i}>a_{p+q+i}>\cdots>b_{(r-1)(p+q)+q+i}, \\
b_{i^{\prime}}>a_{p+i^{\prime}}>b_{p+q+i^{\prime}}>\cdots>a_{(r-1)(p+q)+p+i^{\prime}}>b_{r(p+q)+i^{\prime}} \geq a_{r(p+q)+i}
\end{array}
\end{aligned}
$$

if $a_{r(p+q)+i} \leq b_{r(p+q)+i^{\prime}}$. After the above modification, we can apply Lemma 2.5 to the eigenvalues in the interlacing inequalities with starting terms $a_{i}$ to get a rank $p$ positive semi-definite matrix, and then apply Lemma 2.5 to the eigenvalues in the interlacing inequalities with starting terms $b_{j}$ to get a rank $q$ semi-definite matrix. The result follows.

Following our proof, one can construct the matrices $A$ and $B$ in block diagonal forms as asserted in the last statement of the theorem.

It is easy to use Theorem 2.1 to test whether a given pair of integers $(p, q)$ belongs to $\operatorname{In}(\mathbf{a}, \mathbf{b})$. Here is an example.

Example 2.6 Let $\mathbf{a}=(6,6,4,3,3,2,1)$ and $\mathbf{b}=(5,4,3,3,1,1,1)$. Then the following hold.

(i) $\quad(1,1) \notin \operatorname{In}(\mathbf{a}, \mathbf{b})$ as

$$
\left(b_{1}, \ldots, b_{7-1}\right)-\left(a_{1+1}, \ldots, a_{7}\right)=(5,4,3,3,1,1)-(6,4,3,3,2,1)
$$

has a negative entry.

(ii) $(2,0) \in \operatorname{In}(\mathbf{a}, \mathbf{b})$ as

$$
\begin{aligned}
\left(a_{1}, \ldots, a_{7-0}\right)-\left(b_{1+0}, \ldots, b_{7}\right) & =(6,6,4,3,3,2,1)-(5,4,3,3,1,1,1) \\
& =(1,2,1,0,2,1,0) \\
\left(b_{1}, \ldots, b_{7-2}\right)-\left(a_{2+1}, \ldots, a_{7}\right) & =(5,4,3,3,1)-(4,3,3,2,1)=(1,1,0,1,0) .
\end{aligned}
$$


In fact, if $A=\operatorname{diag}(6,4,6,2,3,3,1)$ and $B=B_{1} \oplus B_{2}$ with

$$
\begin{aligned}
& B_{1}=\left(\begin{array}{cc}
7 / 2 & \sqrt{15} / 2 \\
\sqrt{15} / 2 & 5 / 2
\end{array}\right) \text { and } B_{2}=\left(\begin{array}{cc}
7 / 2 & \sqrt{5} / 2 \\
\sqrt{5} / 2 & 3 / 2
\end{array}\right) \oplus \operatorname{diag}(3,3,1), \\
& \text { then }(A, B) \in \mathcal{H}_{7}(\mathbf{a}) \times \mathcal{H}_{7}(\mathbf{b}) \text { such that } \\
& A-B=\left(\begin{array}{cc}
5 / 2 & -\sqrt{15} / 2 \\
-\sqrt{15} / 2 & 3 / 2
\end{array}\right) \oplus\left[\left(\begin{array}{cc}
5 / 2 & -\sqrt{5} / 2 \\
-\sqrt{5} / 2 & 1 / 2
\end{array}\right) \oplus \operatorname{diag}(0,0,0)\right] \\
& \in \mathcal{H}_{7}(2,0) .
\end{aligned}
$$

We can also test every $(p, q)$ pair of nonnegative integers with $p+q \leq 7$ and depict the set $\operatorname{In}(\mathbf{a}, \mathbf{b})$ as points in $\mathbf{R}^{2}$ as follows.

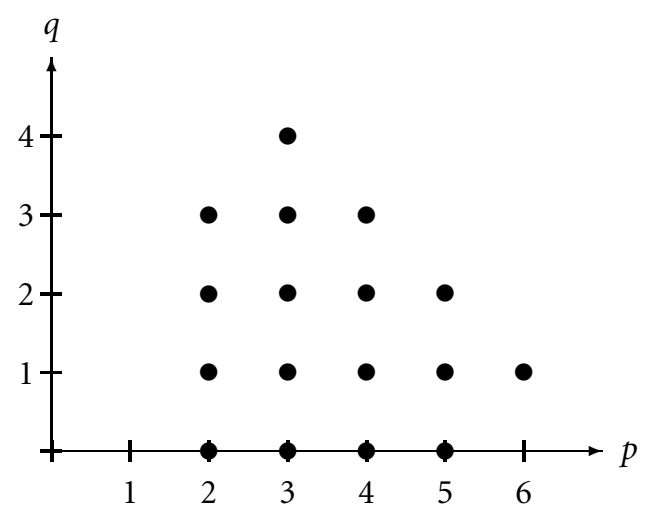

Corollary 2.7 Suppose $\left(p_{1}, q_{1}\right),\left(p_{2}, q_{2}\right) \in \operatorname{In}(\mathbf{a}, \mathbf{b})$. Let $p=\min \left\{p_{1}, p_{2}\right\}$ and $q=\min \left\{q_{1}, q_{2}\right\}$. Then $(p, q) \in \operatorname{In}(\mathbf{a}, \mathbf{b})$.

Proof Suppose $p=p_{i}$ and $q=q_{j}$. Since $\left(p_{1}, q_{1}\right),\left(p_{2}, q_{2}\right) \in \operatorname{In}(\mathbf{a}, \mathbf{b})$, we have

$$
\begin{aligned}
& \mathbf{a}^{n-q_{j}} \geq_{p_{j}} \mathbf{b}_{n-q_{j}} \Rightarrow \mathbf{a}^{n-q} \geq_{p} \mathbf{b}_{n-q}, \\
& \mathbf{b}^{n-p_{i}} \geq_{q_{i}} \mathbf{a}_{n-p_{i}} \Rightarrow \mathbf{b}^{n-p} \geq_{q} \mathbf{a}_{n-p} .
\end{aligned}
$$

Hence, by Theorem 2.1, $(p, q) \in \operatorname{In}(\mathbf{a}, \mathbf{b})$.

\section{A Global Description of $\operatorname{In}(\mathbf{a}, \mathbf{b})$}

While Theorem 2.1 allows us to test if a pair of nonnegative integers lies in $\operatorname{In}(\mathbf{a}, \mathbf{b})$, it would be nice to have a global description of the region for all integer pairs in $\operatorname{In}(\mathbf{a}, \mathbf{b})$. The objective of this section is to obtain such a description.

Note that if $\mathbf{a}$ and $\mathbf{b}$ have a common entry with multiplicities $n_{1}$ and $n_{2}$ in the two vectors such that $n_{1}+n_{2}>n$, then for any $(A, B) \in \mathcal{H}_{n}(\mathbf{a}) \times \mathcal{H}_{n}(\mathbf{b})$, the null space of $A-B$ has dimension at least $n_{1}+n_{2}-m$, and a reduction of the vectors $\mathbf{a}$ and $\mathbf{b}$ is possible in the problem of describing $\operatorname{In}(\mathbf{a}, \mathbf{b})$ as shown in the following proposition. 
Proposition 3.1 Let $\mathbf{a}=\left(a_{1}, \ldots, a_{n}\right)$ and $\mathbf{b}=\left(b_{1}, \ldots, b_{n}\right)$ be two real vectors with entries arranged in descending order. Suppose

$$
a_{i}=a_{i+1}=\cdots=a_{i+n_{1}-1}=b_{j}=b_{j+1}=\cdots=b_{j+n_{2}-1},
$$

for some $i, j, n_{1}, n_{2} \geq 1$ such that $n_{1}+n_{2}>n$. Let $s=n_{1}+n_{2}-n$ and $\mathbf{a}^{\prime}, \mathbf{b}^{\prime}$ be obtained by deleting $s a_{i}$ from each of $\mathbf{a}$ and $\mathbf{b}$. Then $(p, q) \in \operatorname{In}(\mathbf{a}, \mathbf{b})$ if and only if $(p, q) \in \operatorname{In}\left(\mathbf{a}^{\prime}, \mathbf{b}^{\prime}\right)$.

Proof Suppose $A$ and $B$ have eigenvalues $a_{1}, \ldots, a_{n}$ and $b_{1}, \ldots, b_{n}$. Then the intersection of the eigenspaces of $A$ and $B$ associated with $a_{i}$ has dimension $\geq s$. So there exists a unitary $U$ such that $U^{*} A U=A^{\prime} \oplus a_{i} I_{s}$ and $U^{*} B U=B^{\prime} \oplus a_{i} I_{s}$. Therefore, $(p, q) \in \operatorname{In}(\mathbf{a}, \mathbf{b})$ if and only if $(p, q) \in \operatorname{In}\left(\mathbf{a}^{\prime}, \mathbf{b}^{\prime}\right)$.

By the above lemma, to describe $\operatorname{In}(\mathbf{a}, \mathbf{b})$, we can focus on the $(\mathbf{a}, \mathbf{b})$ pair such that $\mathbf{a}$ and $\mathbf{b}$ do not have a common entry whose multiplicities in the two vectors have sum exceeding $n$. To describe the main result in this section, we need the following definition.

Definition 3.2 Suppose $\mathbf{a}=\left(a_{1}, \ldots, a_{n}\right)$ and $\mathbf{b}=\left(b_{1}, \ldots, b_{n}\right)$ are real vectors with entries arranged in descending order. Let

$$
\begin{aligned}
& p_{0}= \begin{cases}n & \text { if } b_{1}<a_{n}, \\
\min \left\{t: 0 \leq t<n, \mathbf{b}^{n-t} \geq \mathbf{a}_{n-t}\right\} & \text { otherwise; }\end{cases} \\
& q_{0}= \begin{cases}n & \text { if } a_{1}<b_{n}, \\
\min \left\{t: 0 \leq t<n, \mathbf{a}^{n-t} \geq \mathbf{b}_{n-t}\right\} & \text { otherwise. }\end{cases}
\end{aligned}
$$

Suppose

$$
\left(a_{1}, \ldots, a_{n}, b_{1}, \ldots, b_{n}\right) \text { has no entry with multiplicity larger than } n \text {. }
$$

Let

$$
\begin{aligned}
& k= \begin{cases}n-p_{0} & \text { if } b_{1} \leq a_{n} \\
\min \left\{t: 0 \leq t<n-p_{0}, \mathbf{b}^{n-p_{0}-t}>\mathbf{a}_{n-p_{0}-t}\right\} & \text { otherwise }\end{cases} \\
& \ell= \begin{cases}n-q_{0} & \text { if } a_{1} \leq b_{n} \\
\min \left\{t: 0 \leq t<n-q_{0}, \mathbf{a}^{n-q_{0}-t}>\mathbf{b}_{n-q_{0}-t}\right\} & \text { otherwise }\end{cases}
\end{aligned}
$$

Furthermore, for $0 \leq i \leq n-\left(p_{0}+q_{0}+\ell\right)$ and $0 \leq j \leq n-\left(p_{0}+q_{0}+k\right)$, let

$Q_{i}$ be the number of positive entries in $\mathbf{b}^{n-p_{i}}-\mathbf{a}_{n-p_{i}}$ with $p_{i}=p_{0}+i$,

$P_{j}$ be the number of positive entries in $\mathbf{a}^{n-q_{j}}-\mathbf{b}_{n-q_{j}}$ with $q_{j}=q_{0}+j$. 
In Example 2.6, we have $(k, \ell)=(1,1)$,

$$
\begin{gathered}
\left(p_{0}, q_{0}\right)=(2,0), \quad\left(p_{0}, Q_{0}\right)=(2,3), \quad\left(P_{0}, q_{0}\right)=(5,0), \\
\left(p_{1}, Q_{1}\right)=(3,4)=\left(P_{4}, q_{4}\right), \quad\left(p_{2}, Q_{2}\right)=(4,3)=\left(P_{3}, q_{3}\right), \\
\left(p_{3}, Q_{3}\right)=(5,2)=\left(P_{2}, q_{2}\right), \quad\left(p_{4}, Q_{4}\right)=(6,1)=\left(P_{1}, q_{1}\right) .
\end{gathered}
$$

In general, we will show in Lemma 3.11 that $p_{k} \leq P_{\ell}$ and $p_{i}+Q_{i}=n=P_{j}+q_{j}$ for all $k \leq i \leq n-\left(p_{0}+q_{0}+\ell\right)$ and $\ell \leq j \leq n-\left(p_{0}+q_{0}+k\right)$. Therefore, the points in

$$
\left\{\left(p_{i}, Q_{i}\right): k \leq i \leq n-\left(p_{0}+q_{0}+\ell\right)\right\} \cup\left\{\left(P_{j}, q_{j}\right): \ell \leq j \leq n-\left(p_{0}+q_{0}+k\right)\right\}
$$

lie on the line segment joining $\left(p_{k}, Q_{k}\right)$ and $\left(P_{\ell}, q_{\ell}\right)$.

Theorem 3.3 Let $\mathbf{a}$ and $\mathbf{b}$ be real vectors satisfying condition (3.3). Use the notation in Definition 3.2 The following conditions hold.

(i) The polygon $\mathcal{P}$ obtained by joining the points

$$
\begin{aligned}
& \left(p_{0}, q_{0}\right),\left(p_{0}, Q_{0}\right),\left(p_{1}, Q_{1}\right), \ldots,\left(p_{k}, Q_{k}\right),\left(P_{\ell}, q_{\ell}\right),\left(P_{\ell-1}, q_{\ell-1}\right), \ldots,\left(P_{0}, q_{0}\right),\left(p_{0}, q_{0}\right) \\
& \text { is convex. } \\
& \text { (ii) In }(\mathbf{a}, \mathbf{b}) \text { consists of all the integer pairs }(p, q) \text { in } \mathcal{P} .
\end{aligned}
$$

In Example 2.6, $\mathcal{P}$ is obtained by joining $(2,0),(2,3),(3,4),(6,1),(5,0),(2,0)$. Before presenting the proof of the theorem, we illustrate how to use the theorem in the following corollaries.

Corollary 3.4 Let $\mathbf{a}$ and $\mathbf{b}$ be real vectors with no common entries. Using the notation in (3.1) and (3.2), we have

$$
\operatorname{In}(\mathbf{a}, \mathbf{b})=\left\{(p, q): p \geq p_{0}, q \geq q_{0}, p+q \leq n\right\}
$$

Proof Since $\mathbf{a}$ and $\mathbf{b}$ have no common entries, we see that for each $i \in\{1, \ldots, k\}$, the vector $\mathbf{b}^{n-p_{i}}-\mathbf{a}_{n-p_{i}}$ is positive, and hence $p_{i}+Q_{i}=n$. Similarly, $P_{j}+q_{j}=n$ for each $j \in\{1, \ldots, \ell\}$. By Theorem 3.3 , the result follows.

Corollary 3.5 Suppose there are $\mu>\nu$ and $0 \leq u, v \leq n$ such that

$$
\mu=a_{1}=\cdots=a_{u}=b_{1}=\cdots=b_{v} \text { and } \nu=a_{u+1}=\cdots=a_{n}=b_{v+1}=\cdots=b_{n} .
$$

Then

$$
\operatorname{In}(\mathbf{a}, \mathbf{b})=\{(u-w, v-w): \max \{0, u+v-n\} \leq w \leq \min \{u, v\}\}
$$

Proof Without loss of generality, we may assume that $u \geq v, \mu=1$ and $\nu=0$. Furthermore, by Proposition 3.1. we may assume that $u+v=n$. Then $\left(p_{0}, q_{0}\right)=$ $(u-v, 0)$. Moreover, $\left(p_{i}, Q_{i}\right)=\left(p_{0}+i, i\right)=\left(P_{i}, q_{i}\right)$ for $i=1, \ldots, v$. By Theorem 3.3. the result follows. 
We establish some lemmas to prove Theorem 3.3. The first three lemmas give additional properties of $p_{0}, q_{0}, P_{i}, Q_{j}$, and confirm that $\left(p_{0}, q_{0}\right),\left(p_{i}, Q_{i}\right),\left(P_{j}, q_{j}\right) \in$ $\operatorname{In}(\mathbf{a}, \mathbf{b})$.

Lemma 3.6 Suppose $\mathbf{a}, \mathbf{b}$ are two real vectors, and $p_{0}, q_{0}$ are defined by (3.1) and (3.2). Then the following conditions hold.

(i) $p_{0}=\min \{p:(p, q) \in \operatorname{In}(\mathbf{a}, \mathbf{b})$ for some $q \geq 0\}$, and $\mathbf{a}^{p_{0}}-\mathbf{b}_{p_{0}}$ is a positive vector if $p_{0}>0$.

(ii) $q_{0}=\min \{q:(p, q) \in \operatorname{In}(\mathbf{a}, \mathbf{b})$ for some $p \geq 0\}$, and $\mathbf{b}^{q_{0}}-\mathbf{a}_{q_{0}}$ is a positive vector if $q_{0}>0$.

(iii) $\left(p_{0}, q_{0}\right) \in \operatorname{In}(\mathbf{a}, \mathbf{b})$.

Proof (i) Suppose $p_{0}$ is given by (3.1). If $p_{0}=n$, then $b_{1}<a_{n}$ and $\operatorname{In}(\mathbf{a}, \mathbf{b})=$ $\{(n, 0)\}$. If $p_{0}<n$, then we have $b_{j} \geq a_{p_{0}+j}$ for all $1 \leq j \leq n-p_{0}$. Let $A=\operatorname{diag}\left(a_{1}, \ldots, a_{n}\right)$ and $B=\operatorname{diag}\left(b_{n-p_{0}+1}, \ldots, b_{n}, b_{1}, \ldots, b_{n-p_{0}}\right)$. Then $A-B$ has at most $p_{0}$ positive eigenvalues. Therefore,

$$
p_{0} \geq \min \{p:(p, q) \in \operatorname{In}(\mathbf{a}, \mathbf{b}) \text { for some } q \geq 0\} .
$$

On the other hand, suppose $(p, q) \in \operatorname{In}(\mathbf{a}, \mathbf{b})$ for some $q \geq 0$. Then there exists $(A, B) \in \mathcal{H}_{n}(\mathbf{a}) \times \mathcal{H}_{n}(\mathbf{b})$ such that $A-B \in \mathcal{H}_{n}(p, q)$. By Theorem 2.1, we have $\mathbf{b}^{n-p} \geq \mathbf{a}_{n-p}$. Therefore, $p \geq p_{0}$. Hence,

$$
p_{0} \leq \min \{p:(p, q) \in \operatorname{In}(\mathbf{a}, \mathbf{b}) \text { for some } q \geq 0\} .
$$

If $p_{0}>0$, then there exists $1 \leq i \leq n-\left(p_{0}-1\right)$ such that $a_{p_{0}-1+i}>b_{i}$. So for all $1 \leq j \leq p_{0}$ we have $a_{j} \geq a_{p_{0}-1+i}>b_{i} \geq b_{n-p_{0}+j}$, i.e., $\mathbf{a}^{p_{0}}-\mathbf{b}_{p_{0}}$ is positive. This proves (i). The proof of (ii) is similar.

(iii) By the results in (i) and (ii), we can choose $p \geq p_{0}$ and $q \geq q_{0}$ such that $\left(p, q_{0}\right)$ and $\left(p_{0}, q\right) \in \operatorname{In}(\mathbf{a}, \mathbf{b})$. Hence, by Corollary 2.7 $\left(p_{0}, q_{0}\right) \in \operatorname{In}(\mathbf{a}, \mathbf{b})$.

Note that assumption (3.3) is not needed in Lemma 3.6 .

Lemma 3.7 Suppose $\mathbf{a}$ and $\mathbf{b}$ are real vectors satisfying condition (3.3). Let $s \in$ $\{0, \ldots, n-1\}$ be such that $\mathbf{b}^{n-s}-\mathbf{a}_{n-s}$ has a non-positive entry. Then $\mathbf{a}^{s+1}-\mathbf{b}_{s+1}$ is positive.

Proof Suppose the conclusion is not true. Then $\mathbf{a}^{s+1}-\mathbf{b}_{s+1}$ is not positive. Hence there is $i \in\{1, \ldots, s+1\}$ such that $a_{i} \leq b_{n-s-1+i}$. Since the vector $\mathbf{b}^{n-s}-\mathbf{a}_{n-s}$ has a non-positive entry, $b_{j} \leq a_{s+j}$ for some $j \in\{1, \ldots, n-s\}$. Hence

$$
b_{j}=a_{s+j} \leq a_{s+j-1} \leq \cdots \leq a_{i} \leq b_{n-s-1+i} \leq \cdots \leq b_{j} .
$$

Consequently, all the inequalities become equalities, and the multiplicity of $a_{i}=b_{j}$ in the vector $\left(a_{1}, \ldots, a_{n}, b_{1}, \ldots, b_{n}\right)$ equals $(s+j-i+1)+(n-s+i-j)=n+1$, contradicting assumption (3.3). 
By Lemma 3.7 and the definition of $\ell$ and $k$, we see that

$$
\left(n-q_{0}-\ell, q_{0}+\ell\right),\left(n-p_{0}-k, p_{0}+k\right) \in \operatorname{In}(\mathbf{a}, \mathbf{b})
$$

if $\mathbf{a}$ and $\mathbf{b}$ satisfy 3.3 .

Lemma 3.8 Let $\mathbf{a}$ and $\mathbf{b}$ be real vectors satisfying (3.3). Use the notation in Definition 3.2 For $0 \leq i \leq n-\left(p_{0}+q_{0}+\ell\right)$ and $0 \leq j \leq n-\left(p_{0}+q_{0}+k\right)$, we have

(i) $\mathbf{a}^{p_{0}+i}>\mathbf{b}_{p_{0}+i}$ and $\mathbf{b}^{q_{0}+j}>\mathbf{a}_{q_{0}+j}$.

(ii) $\left(p_{i}, Q_{i}\right),\left(P_{j}, q_{j}\right) \in \operatorname{In}(\mathbf{a}, \mathbf{b})$.

(iii) $Q_{i}=\max \left\{q:\left(p_{0}+i, q\right) \in \operatorname{In}(\mathbf{a}, \mathbf{b})\right\}$ and $P_{j}=\max \left\{p:\left(p, q_{0}+j\right) \in \operatorname{In}(\mathbf{a}, \mathbf{b})\right\}$.

(iv) $p_{0}+q_{0}+k+\ell \leq n$.

Proof If $p_{0}$ or $q_{0}=n$, then $k=\ell=0$, and the results follow. Therefore, in the rest of the proof, we assume that $p_{0}, q_{0}<n$.

(i) It follows from the definitions of $\ell$ and $k$ that $\mathbf{a}^{n-q_{0}-\ell}>\mathbf{b}_{n-q_{0}-\ell}$ and $\mathbf{b}^{n-p_{0}-k}>$ $\mathbf{a}_{n-p_{0}-k}$. For $0 \leq i \leq n-\left(p_{0}+q_{0}+\ell\right)$ we have $p_{0}+i \leq n-q_{0}-\ell$. Therefore, $\mathbf{a}^{p_{0}+i}>\mathbf{b}_{p_{0}+i}$. Similarly, $\mathbf{b}^{q_{0}+j}>\mathbf{a}_{q_{0}+j}$ for $0 \leq n-\left(p_{0}+q_{0}+k\right)$.

(ii) Since, $\operatorname{diag}\left(a_{1}, \ldots, a_{n}\right)-\operatorname{diag}\left(b_{n-p_{i}+1}, \ldots, b_{n}, b_{1}, \ldots, b_{n-p_{i}}\right) \in \mathcal{H}_{n}\left(p_{i}, Q_{i}\right)$, we have $\left(p_{i}, Q_{i}\right) \in \operatorname{In}(\mathbf{a}, \mathbf{b})$. Similarly, $\left(P_{j}, q_{j}\right) \in \operatorname{In}(\mathbf{a}, \mathbf{b})$.

(iii) Suppose $\left(p_{i}, q\right) \in \operatorname{In}(\mathbf{a}, \mathbf{b})$. Then $\mathbf{b}^{n-p_{i}} \geq_{q} \mathbf{a}_{n-p_{i}}$. So, $q \leq Q_{i}$. Hence,

$$
Q_{i}=\max \left\{q:\left(p_{0}+i, q\right) \in \operatorname{In}(\mathbf{a}, \mathbf{b})\right\} .
$$

Similarly, we have

$$
P_{j}=\max \left\{p:\left(p, q_{0}+j\right) \in \operatorname{In}(\mathbf{a}, \mathbf{b})\right\} .
$$

(iv) Since $\left(n-q_{0}-\ell, q_{0}+\ell\right) \in \operatorname{In}(\mathbf{a}, \mathbf{b})$, we have $n-q_{0}-\ell \geq p_{0}$ by Lemma 3.6 From the definition of $k$ and $\mathbf{a}^{n-q_{0}-\ell}>\mathbf{b}_{n-q_{0}-\ell}$, we have $n-q_{0}-\ell \geq p_{0}+k$. Thus, $p_{0}+q_{0}+k+\ell \leq n$.

Clearly, $P_{j}$ is equal to $n-q_{j}$ minus the number of zero entries in $\mathbf{a}^{n-q_{j}}-\mathbf{b}_{n-q_{j}}$. Therefore, in order to study the relationship between $P_{j}$ and $P_{j+1}$, we need to keep track of the zero entries in the vector $\mathbf{a}^{n-q_{j}}-\mathbf{b}_{n-q_{j}}$ and investigate how they are related to the entries of $\mathbf{a}^{n-q_{j}-1}-\mathbf{b}_{n-q_{j}-1}$. For this reason, we introduce the following definition.

Definition 3.9 For $1 \leq i \leq j \leq m \leq n$, we say that $[i, j]=\{t: i \leq t \leq j\}$ is a maximal interval of $\left(\mathbf{a}^{m}, \mathbf{b}_{m}\right)$ if

$$
a_{i-1}>a_{i}=a_{i+1}=\cdots=a_{j}=b_{n-m+i}=b_{n-m+i+1}=\cdots=b_{n-m+j}>b_{n-m+j+1} .
$$

The length of a maximal interval $[i, j]$ is given by $j-i+1$. The set of all maximal intervals of $\left(\mathbf{a}^{m}, \mathbf{b}_{m}\right)$ will be denoted by $S\left(\mathbf{a}^{m}, \mathbf{b}_{m}\right)$. Let $T=T\left(\mathbf{a}^{m}, \mathbf{b}_{m}\right)$ be the maximum length of a maximal interval of $\left(\mathbf{a}^{m}, \mathbf{b}_{m}\right)$. For $1 \leq t \leq T$, let $s_{t}$ be the number of maximal intervals of $\left(\mathbf{a}^{m}, \mathbf{b}_{m}\right)$ with length $t$. The sequence $\left(s_{1}, s_{2}, \ldots, s_{T}\right)$ will be denoted by $\mathbf{s}\left(\mathbf{a}^{m}, \mathbf{b}_{m}\right)$. 
Lemma 3.10 Suppose $\mathbf{a}^{m} \geq \mathbf{b}_{m}$ for some $1 \leq m \leq n$. Then the following conditions hold.

(i) $\mathbf{a}^{m}>_{q} \mathbf{b}_{m}$ where $q=m-\sum_{t=1}^{T} t s_{t}$.

(ii) $[i, j] \in S\left(\mathbf{a}^{m-1}, \mathbf{b}_{m-1}\right)$ if and only if $[i, j+1] \in S\left(\mathbf{a}^{m}, \mathbf{b}_{m}\right)$.

(iii) $\mathbf{a}^{m-1}>_{q_{1}} \mathbf{b}_{m-1}$, where $q_{1}=q-1+\sum_{t=1}^{T} s_{t}$.

(iv) If $\mathbf{a}^{m-2}>_{q_{2}} \mathbf{b}_{m-2}$, then $q_{2}-q_{1} \leq q_{1}-q$.

Here, we assume that $m>1$ for (ii)-(iii) and $m>2$ for (iv).

Proof Condition (i) holds because $\sum_{t=1}^{T} t s_{t}$ is the number of zero entries in $\mathbf{a}^{m}-\mathbf{b}_{m}$. To prove (ii), suppose $[i, j] \in S\left(\mathbf{a}^{m-1}, \mathbf{b}_{m-1}\right)$. Then we have

$$
\begin{aligned}
a_{i-1}>a_{i} & =a_{i+1}=\cdots=a_{j} \\
& =b_{n-(m-1)+i}=b_{n-(m-1)+i+1}=\cdots=b_{n-(m-1)+j}>b_{n-(m-1)+j+1} .
\end{aligned}
$$

Since

$$
\begin{gathered}
a_{i-1}>a_{i} \geq b_{n-m+i} \geq b_{n-m+i+1}=a_{i}, \\
a_{j} \geq a_{j+1} \geq b_{n-m+j+1}=a_{j}>b_{n-(m-1)+j+1},
\end{gathered}
$$

we have $a_{i}=b_{n-m+i}=b_{n-m+i+1}$ and $a_{j}=a_{j+1}=b_{n-m+j+1}$. This gives

$$
\begin{aligned}
a_{i-1}>a_{i} & =a_{i+1}=\cdots=a_{j+1} \\
& =b_{n-m+i}=b_{n-m+i+1}=\cdots=b_{n-m+j+1}>b_{n-m+j+2} .
\end{aligned}
$$

Thus, $[i, j+1] \in S\left(\mathbf{a}^{m}, \mathbf{b}_{m}\right)$. Conversely, if $[i, j+1] \in S\left(\mathbf{a}^{m}, \mathbf{b}_{m}\right)$ for some $j \geq i$, then (3.5) holds. Thus (3.4) follows and $[i, j] \in S\left(\mathbf{a}^{m-1}, \mathbf{b}_{m-1}\right)$.

To prove (iii), let $\mathbf{s}\left(\mathbf{a}^{m}, \mathbf{b}_{m}\right)=\left(s_{1}, s_{2}, \ldots, s_{T}\right)$. Then it follows from (ii) that $\mathbf{s}\left(\mathbf{a}^{m-1}, \mathbf{b}_{m-1}\right)=\left(s_{2}, s_{3}, \ldots, s_{T}\right)$. Hence,

$$
q_{1}=m-1-\sum_{t=2}^{T}(t-1) s_{t}=m-1-\sum_{t=1}^{T} t s_{t}+\sum_{t=1}^{T} s_{t}=q-1+\sum_{t=1}^{T} s_{t} .
$$
(iv).

From (iii) we have $q_{2}-q_{1}=\sum_{t=2}^{T} s_{t}-1 \leq \sum_{t=1}^{T} s_{t}-1=q_{1}-q$. This proves

Applying Lemma 3.10 to the quantities in Definition 3.2, we readily deduce the following.

Lemma 3.11 Using the notation in Definitions 3.2 and 3.9 the following conditions hold.

(i) $k=T\left(\mathbf{b}_{n-p_{0}}, \mathbf{a}_{n-p_{0}}\right), \ell=T\left(\mathbf{a}_{n-q_{0}}, \mathbf{b}_{n-q_{0}}\right)$. 
(ii) Suppose $\mathbf{s}\left(\mathbf{b}_{n-p_{0}}, \mathbf{a}_{n-p_{0}}\right)=\left(s_{1}, s_{2}, \ldots, s_{k}\right)$ and $\mathbf{s}\left(\mathbf{a}_{n-q_{0}}, \mathbf{b}_{n-q_{0}}\right)=\left(s_{1}^{\prime}, s_{2}^{\prime}, \ldots, s_{\ell}^{\prime}\right)$. Then

$$
\begin{aligned}
& Q_{i+1}=Q_{i}-1+\sum_{t=i+1}^{k} s_{t} \quad \text { for } 0 \leq i<k, \\
& P_{j+1}=P_{j}-1+\sum_{t=j+1}^{k} s_{t}^{\prime} \quad \text { for } 0 \leq j<\ell .
\end{aligned}
$$

(iii) For $k \leq i<n-\left(p_{0}+q_{0}+\ell\right)$ and $\ell \leq j<n-\left(p_{0}+q_{0}+k\right)$, we have

$$
Q_{i+1}=Q_{i}-1 \text { and } P_{j+1}=P_{j}-1 .
$$

Moreover, for $k \leq i \leq n-\left(p_{0}+q_{0}+\ell\right)$ and $\ell \leq j \leq n-\left(p_{0}+q_{0}+k\right)$, we have

$$
p_{i}+Q_{i}=n=P_{j}+q_{j}
$$

(iv) For $0<i<n-\left(p_{0}+q_{0}+\ell\right)$ and $\ell<j<n-\left(p_{0}+q_{0}+k\right)$, we have

$$
Q_{i}-Q_{i-1} \geq Q_{i+1}-Q_{i} \quad \text { and } \quad P_{j}-P_{j-1} \geq P_{j+1} \geq P_{j+1}-P_{j}
$$

Proof of Theorem 3.3 (i) From $\left(p_{0}, q_{0}\right)$ to $\left(p_{0}, Q_{0}\right)$, we have a vertical straight line segment. Note that the slope of the line segment from $\left(p_{i-1}, Q_{i-1}\right)$ to $\left(p_{i}, Q_{i}\right)$ equals $Q_{i}-Q_{i-1}$, and the slope of the line segment from $\left(p_{i}, Q_{i}\right)$ to $\left(p_{i+1}, Q_{i+1}\right)$ is $Q_{i+1}-Q_{i}$. By Lemma 3.11(iv), we see that $Q_{i}-Q_{i-1} \geq Q_{i+1}-Q_{i}$. Thus, the polygonal curve joining the points $\left(p_{0}, Q_{0}\right),\left(p_{1}, Q_{1}\right), \ldots,\left(p_{k}, Q_{k}\right)$ is convex. The line segment joining $\left(p_{k}, Q_{k}\right)$ and $\left(P_{\ell}, q_{\ell}\right)$ is a line segment with negative slope. Finally, the polygonal curve joining the points $\left(p_{0}, q_{0}\right),\left(P_{0}, q_{0}\right), \ldots,\left(P_{\ell}, q_{\ell}\right)$ is concave by Lemma 3.11 (iv). Thus $\mathcal{P}$ is a convex subset contained in the set

$$
\left\{(p, q): p_{0} \leq p \leq n-q_{\ell}, q_{0} \leq q \leq n-p_{k} \text {, and } p+q \leq n\right\} .
$$

(ii) Suppose $(p, q) \in \mathcal{P}$. Let $p=p_{i}$ and $q=q_{j}$ for some $0 \leq i \leq n-\left(p_{0}+q_{0}+\ell\right)$ and $0 \leq j \leq n-\left(p_{0}+q_{0}+k\right)$. Then $p_{i} \leq P_{j}$ and $q_{j} \leq Q_{i}$. Since $\left(p_{i}, Q_{i}\right)$ and $\left(P_{j}, q_{j}\right) \in \operatorname{In}(\mathbf{a}, \mathbf{b})$. By Corollary 2.7 $\left(p_{i}, q_{j}\right) \in \operatorname{In}(\mathbf{a}, \mathbf{b})$.

Conversely, suppose $(p, q) \in \operatorname{In}(\mathbf{a}, \mathbf{b})$. By Theorem 3.6, we have $p \geq p_{0}, q \geq q_{0}$ and $p+q \leq n$. Let $p=p_{i}$ and $q=q_{j}$ for some $i, j \geq 0$. If $i>n-\left(p_{0}+q_{0}+\ell\right)$, then we have

$$
q_{j} \leq n-p_{i}<q_{0}+\ell \quad \Rightarrow \quad p_{i} \leq P_{j} \leq n-q_{\ell} \quad \Rightarrow \quad i \leq n-\left(p_{0}+q_{0}+\ell\right),
$$

which is a contradiction. Therefore, $0 \leq i \leq n-\left(p_{0}+q_{0}+\ell\right)$. Similarly, we have $0 \leq j \leq n-\left(p_{0}+q_{0}+k\right)$. Since $\left(p_{i}, q_{j}\right) \in \operatorname{In}(\mathbf{a}, \mathbf{b})$, we have $p_{i} \leq P_{j}$ and $q_{j} \leq Q_{i}$ by Lemma 3.8. If either $p_{i}=P_{j}$ or $q_{j}=Q_{i}$, then $(p, q) \in \mathcal{P}$. So we may assume that $p_{i}<P_{j}$ and $q_{j}<Q_{i}$. Consider the positive numbers

$$
t_{1}=j\left(P_{j}-p_{i}\right), \quad t_{2}=i\left(Q_{i}-q_{j}\right), \quad \text { and } \quad t_{3}=\left(P_{j}-p_{i}\right)\left(Q_{i}-q_{j}\right) .
$$


Then by direct computation we have

$$
\begin{aligned}
\frac{t_{1}\left(p_{i}, Q_{i}\right)+t_{2}\left(P_{j}, q_{j}\right)+t_{3}\left(p_{0}, q_{0}\right)}{t_{1}+t_{2}+t_{3}} & =\frac{\left(t_{1} p_{i}+t_{2} P_{j}+t_{3} p_{0}, t_{1} Q_{i}+t_{2} q_{j}+t_{3} q_{0}\right)}{t_{1}+t_{2}+t_{3}} \\
& =\left(p_{i}, q_{j}\right) .
\end{aligned}
$$

Thus, $(p, q)$ lies in $\mathcal{P}$.

\section{Elements in $\operatorname{In}(\mathbf{a}, \mathbf{b})$ Attainable by Diagonal Matrices}

In this section, we determine those elements in $\operatorname{In}(\mathbf{a}, \mathbf{b})$ that are attainable by diagonal matrices. Clearly, if $A$ and $B$ are diagonal matrices with eigenvalues so that the eigenvalues of $A$ and those of $B$ are mutually distinct, then $A-B$ is invertible. If $A$ and $B$ have $m$ common eigenvalues (counting multiplicities), then $A-B$ has at most $m$ zero eigenvalues. It turns out that this is the only additional restriction on $(p, q) \in \operatorname{In}(\mathbf{a}, \mathbf{b})$ to be attainable by diagonal matrices.

Theorem 4.1 Suppose $\mathbf{a}$ and $\mathbf{b}$ have $m$ common entries counting multiplicities. Then there are diagonal matrices $A \in \mathcal{H}_{n}(\mathbf{a})$ and $B \in \mathcal{H}_{n}(\mathbf{b})$ such that $A-B \in \mathcal{H}_{n}(p, q)$ if and only if $(p, q) \in \operatorname{In}(\mathbf{a}, \mathbf{b})$ and $p+q \geq n-m$.

To prove Theorem 4.1 we need the following.

Lemma 4.2 Let $\mathbf{a}=\left(a_{1}, a_{2}, \ldots, a_{n}\right)$ and $\mathbf{b}=\left(b_{1}, b_{2}, \ldots, b_{n}\right) \in \mathbf{R}^{n}$ with

$$
a_{1} \geq a_{2} \geq \cdots \geq a_{n} \text { and } b_{1} \geq b_{2} \geq \cdots \geq b_{n} .
$$

Given $1 \leq j_{1} \leq i_{1} \leq n$, let $\hat{\mathbf{a}}$ and $\hat{\mathbf{b}}$ be obtained from $\mathbf{a}$ and $\mathbf{b}$ by deleting $a_{i_{1}}$ and $b_{j_{1}}$ from $\mathbf{a}$ and $\mathbf{b}$, respectively. Suppose $\mathbf{a}>_{p} \mathbf{b}$ for some $0 \leq p \leq n$. We have

(i) $\hat{\mathbf{a}} \geq \hat{\mathbf{b}}$.

(ii) If $1 \leq p \leq n$, then $\hat{\mathbf{a}} \geq_{p-1} \hat{\mathbf{b}}$.

(iii) If $a_{i}=b_{i}$ for some $j_{1} \leq i \leq i_{1}$, then $\hat{\mathbf{a}} \geq_{p} \hat{\mathbf{b}}$.

Proof Since

$$
\hat{a}_{i}=\left\{\begin{array}{ll}
a_{i} & \text { if } 1 \leq i<i_{1}, \\
a_{i+1} & \text { if } i_{1} \leq i \leq n-1,
\end{array} \quad \hat{b}_{j}= \begin{cases}b_{j} & \text { if } 1 \leq j<j_{1}, \\
b_{j+1} & \text { if } j_{1} \leq j \leq n-1,\end{cases}\right.
$$

we have

$$
\begin{array}{lll}
1 \leq i<j_{1} \quad & \Rightarrow \quad \hat{a}_{i}=a_{i} \geq b_{i}=\hat{b}_{i} \\
j_{1} \leq i<i_{1} \quad \Rightarrow \quad & \hat{a}_{i}=a_{i} \geq b_{i} \geq b_{i+1}=\hat{b}_{i} \\
i_{1} \leq i<n & \Rightarrow & \hat{a}_{i}=a_{i+1} \geq b_{i+1}=\hat{b}_{i}
\end{array}
$$

and (i) holds. 
Note that every strict inequality $a_{i}>b_{i}$ for $1 \leq i<i_{1}$ (or $i_{1}<i \leq n$ ) gives a strict inequality $\hat{a}_{i}>\hat{b}_{i}\left(\right.$ or $\left.\hat{a}_{i-1}>\hat{b}_{i-1}\right)$. This proves (ii) and the case when $i=i_{1}$ or $j_{1}$ in (iii).

For (iii), we may assume that $a_{i_{1}}>b_{i_{1}}$ and $i_{1}>j_{1}$. Note that

$$
\begin{aligned}
\left(\hat{a}_{1}, \hat{a}_{2}, \ldots, \hat{a}_{j_{1}-1}\right) & =\left(a_{1}, a_{2}, \ldots, a_{j_{1}-1}\right), \\
\left(\hat{b}_{1}, \hat{b}_{2}, \ldots, \hat{a}_{j_{1}-1}\right) & =\left(b_{1}, b_{2}, \ldots, b_{j_{1}-1}\right), \\
\left(\hat{a}_{j_{1}}, \hat{a}_{j_{1}+1}, \ldots, \hat{a}_{i_{1}-1}\right) & =\left(a_{j_{1}}, a_{j_{1}+1}, \ldots, a_{i_{1}-1}\right), \\
\left(\hat{b}_{j_{1}}, \hat{b}_{j_{1}+1}, \ldots, \hat{b}_{i_{1}-1}\right) & =\left(b_{j_{1}+1}, b_{j_{1}+2}, \ldots, b_{i_{1}}\right), \\
\left(\hat{a}_{i_{1}}, \hat{a}_{i_{1}+1}, \ldots, \hat{a}_{n-1}\right) & =\left(a_{i_{1}+1}, a_{i_{1}+2}, \ldots, a_{n}\right), \\
\left(\hat{b}_{i_{1}}, \hat{b}_{i_{1}+1}, \ldots, \hat{b}_{n-1}\right) & =\left(b_{i_{1}+1}, b_{i_{1}+2}, \ldots, a_{n}\right) .
\end{aligned}
$$

Apply Lemma 3.10 (iii) to $\left(a_{j_{1}}, a_{j_{1}+1}, \ldots, a_{i_{1}}\right)$ and $\left(b_{j_{1}}, b_{j_{1}+2}, \ldots, b_{i_{1}}\right)$; by the fact that at least one $s_{k}$ is positive, we can conclude that the number of strict inequalities in $\left(\hat{a}_{j_{1}}, \hat{a}_{j_{1}+1}, \ldots, \hat{a}_{i_{1}-1}\right)-\left(\hat{b}_{j_{1}}, \hat{b}_{j_{1}+1}, \ldots, \hat{b}_{i_{1}-1}\right)$ is no less than that of

$$
\left(a_{j_{1}}, a_{j_{1}+1}, \ldots, a_{i_{1}}\right)-\left(b_{j_{1}}, b_{j_{1}+2}, \ldots, b_{i_{1}}\right) .
$$

Therefore, the number of entries in $\hat{\mathbf{a}}-\hat{\mathbf{b}}$ is no less than that of $\mathbf{a}-\mathbf{b}$.

Proof of Theorem 4.1 Suppose $A$ and $B$ are diagonal matrices with eigenvalues $a_{1}, \ldots, a_{n}$ and $b_{1}, \ldots, b_{n}$ such that $A-B \in \mathcal{H}_{n}(p, q)$. So, $(p, q) \in \operatorname{In}(\mathbf{a}, \mathbf{b})$. Also, the number of zero diagonal entries is at most $m$. Therefore, $m \geq n-p-q$. Hence, $p+q \geq n-m$.

We prove the converse by induction on $m$. Let $(p, q) \in \operatorname{In}(\mathbf{a}, \mathbf{b})$ and $p+q \geq n-m$. If $p+q=n$. Then the result follows from Theorem 2.1. So the result holds for $m=0$ and we may assume that $n>p+q$.

Let $m>0$. Assume the result holds whenever $\mathbf{a}$ and $\mathbf{b}$ have $m-1$ entries in common. Suppose $\mathbf{a}$ and $\mathbf{b}$ have $m$ common entries and $(p, q) \in \operatorname{In}(\mathbf{a}, \mathbf{b})$, with $p+q \geq n-m$. By Theorem 2.1, we have $\mathbf{a}^{n-q} \geq_{p} \mathbf{b}_{n-q}$ and $\mathbf{b}^{n-p} \geq_{q} \mathbf{a}_{n-p}$. We may assume that $n>p+q \geq n-m$. We are going to show that we can delete common entries from $\mathbf{a}$ and $\mathbf{b}$ to obtain vectors $\hat{\mathbf{a}}$ and $\hat{\mathbf{b}} \in \mathbf{R}^{n-1}$ so that $\hat{\mathbf{a}}^{n-1-q} \geq_{p} \hat{\mathbf{b}}_{n-1-q}$ and $\hat{\mathbf{b}}^{n-1-p} \geq_{q} \hat{\mathbf{a}}_{n-1-p}$. Since $\hat{\mathbf{a}}$ and $\hat{\mathbf{b}}$ have only $m-1$ entries in common and $p+q \geq(n-1)-(m-1)$, the result will follow.

Consider the following cases:

Case 1: $\mathbf{a}^{n-q} \geq_{p+1} \mathbf{b}_{n-q}$ and $\mathbf{b}^{n-p} \geq_{q+1} \mathbf{a}_{n-p}$.

Since $m>0$, we can choose $i_{1}=\min \left\{i: a_{i}=b_{j}\right.$ for some $\left.j\right\}$ and $j_{1}=\min \{j$ : $\left.b_{j}=a_{i_{1}}\right\}$. Let $\hat{\mathbf{a}}$ and $\hat{\mathbf{b}}$ be obtained from $\mathbf{a}$ and $\mathbf{b}$ by deleting $a_{i_{1}}$ and $b_{j_{1}}$ respectively.

If $i_{1}>n-q$, then $\hat{\mathbf{a}}^{n-1-q}=\mathbf{a}^{n-1-q}$. Therefore, $\hat{\mathbf{a}}^{n-1-q} \geq_{p} \hat{\mathbf{b}}_{n-1-q}$.

If $i_{1} \leq n-q$, then $b_{j_{1}-1}>b_{j_{1}}=a_{i_{1}} \geq b_{q+i_{1}}$ and we have $q+i_{1} \geq j_{1}$. By Lemma 4.2 (ii), $\hat{\mathbf{a}}^{n-1-q} \geq_{p} \hat{\mathbf{b}}_{n-1-q}$.

Similarly, we have $\hat{\mathbf{b}}^{n-1-p} \geq_{q} \hat{\mathbf{a}}_{n-1-p}$. 
Case 2: $\mathbf{a}^{n-q}>_{p} \mathbf{b}_{n-q}$.

Since $n-q>p$, let $i_{1}=\min \left\{t: 1 \leq t \leq n-q\right.$ and $\left.a_{t}=b_{q+t}\right\} \leq p+1$. Let $\hat{\mathbf{a}}$ and $\hat{\mathbf{b}}$ be obtained from $\mathbf{a}$ and $\mathbf{b}$ by deleting $a_{i_{1}}$ and $b_{q+i_{1}}$, respectively. Then $\hat{\mathbf{a}}$ and $\hat{\mathbf{b}}$ have $m-1$ entries in common. By Lemma 4.2 (iii), $\hat{\mathbf{a}}^{n-1-q} \geq_{p} \hat{\mathbf{b}}_{n-1-q}$. Consider the following cases.

Subcase 2a: If $\mathbf{b}^{n-p} \geq_{q+1} \mathbf{a}_{n-p}$, then it follow from Lemma4.2(ii) that $\hat{\mathbf{b}}^{n-1-p} \geq_{q}$ $\hat{\mathbf{a}}_{n-1-p}$.

Subcase 2b: If $\mathbf{b}^{n-p}>_{q} \mathbf{a}_{n-p}$, then

$$
\min \left\{s: 1 \leq s \leq n-p \text { and } b_{s}=a_{p+s}\right\} \leq q+1 \leq q+i_{1} .
$$

It follows from Lemma 4.2 (iii) that $\hat{\mathbf{b}}^{n-1-p} \geq_{q} \hat{\mathbf{a}}_{n-1-p}$.

\section{Ranks and Multiple Eigenvalues}

By Theorem 3.3, we can determine the set $R(\mathbf{a}, \mathbf{b})$ of all possible ranks for a matrix of the form $A-B$ with $(A, B) \in \mathcal{H}_{n}(\mathbf{a}) \times \mathcal{H}_{n}(\mathbf{b})$. Evidently, we have

$$
R(\mathbf{a}, \mathbf{b})=\{p+q:(p, q) \in \operatorname{In}(\mathbf{a}, \mathbf{b})\} .
$$

Nevertheless, it is interesting that the result can be put into the following simple form.

Theorem 5.1 Let $\mathbf{a}, \mathbf{b}$ be real vectors, and define $p_{0}$ and $q_{0}$ as in (3.1) and (3.2). Let $m$ be the largest multiplicity of an entry in $\left(a_{1}, \ldots, a_{n}, b_{1}, \ldots, b_{n}\right)$ and let $r=$ $\min \{2 n-m, n\}$. Suppose $R(\mathbf{a}, \mathbf{b})$ is the set of rank values of matrices of the form $A-B$, where $(A, B) \in \mathcal{H}_{n}(\mathbf{a}) \times \mathcal{H}_{n}(\mathbf{b})$. Then one of the following holds.

(i) There exist real numbers $\mu>\nu$ and $u, v \in\{0, \ldots, n\}$ such that

$$
\mathbf{a}=(\underbrace{\mu, \ldots, \mu}_{u}, \nu, \ldots, \nu), \quad \mathbf{b}=(\underbrace{\mu, \ldots, \mu}_{v}, \nu, \ldots, \nu),
$$

and

$$
R(\mathbf{a}, \mathbf{b})=\{u+v-2 j: \max \{0, u+v-n\} \leq j \leq \min \{u, v\}\} .
$$

(ii) Condition (i) does not hold, $\mathbf{a}=\mathbf{b}$, and $R(\mathbf{a}, \mathbf{b})=\{0\} \cup\{2, \ldots, r\}$.

(iii) Conditions (i) and (ii) do not hold, and $R(\mathbf{a}, \mathbf{b})=\left\{p_{0}+q_{0}, \ldots, r\right\}$.

Moreover, if $t \in R(\mathbf{a}, \mathbf{b})$, then there are block diagonal matrices

$$
A=A_{1} \oplus \cdots \oplus A_{t} \in \mathcal{H}_{n}(\mathbf{a}) \quad \text { and } \quad B=B_{1} \oplus \cdots \oplus B_{t} \in \mathcal{H}_{n}(\mathbf{b})
$$

with the same block sizes such that $A_{j}-B_{j}$ has rank one for $j=1, \ldots, t$.

Note that in the theorem, we include the case when $\left(a_{1}, \ldots, a_{n}, b_{1}, \ldots, b_{n}\right)$ has an entry with multiplicity larger than $n$. 
Proof (1) Suppose a, b satisfy the condition in (i). The result follows from Corollary 3.5 .

(2) Suppose condition (i) does not hold and $\mathbf{a}=\mathbf{b}$. If $A=B=\operatorname{diag}\left(a_{1}, \ldots, a_{n}\right)$, then $A-B \in \mathcal{H}_{n}(0,0)$. Since $A$ and $B$ have the same trace, we see that $A-B$ cannot have rank 1 .

Without loss of generality, we may assume that $r=n$. We prove the following claim by induction on $n$ :

There are matrices $A, B \in \mathcal{H}_{n}(\mathbf{a})$ such that $A-B \in \mathcal{H}_{n}(p, q)$ whenever $2 \leq p+q \leq$ $n$ with $p=q$ or $p=q+1$.

The claim is clear if $n=3,4$. Suppose $n \geq 5$ and $2 \leq p+q \leq n$ with $p=q$ or $p=q+1$. Since a has at least three distinct entries, each entry has multiplicity at most $n / 2$. Suppose $a_{r}>a_{s}$, where $a_{r}, a_{s}$ have the two largest multiplicities in the vector $\mathbf{a}$.

For $2 \leq p+q \leq 3$, choose $a_{w} \notin\left\{a_{u}, a_{v}\right\}$ and let $A_{1}=\operatorname{diag}\left(a_{u}, a_{v}, a_{w}\right)$. Then there exists a diagonal matrix $B_{1}$ with the same eigenvalues as $A_{1}$ and $A_{1}-B_{1} \in \mathcal{H}_{3}(p, q)$. Remove $a_{u}, a_{v}, a_{w}$ from a to get $\mathbf{a}^{\prime}$. Then $A_{1} \oplus \operatorname{diag}\left(\mathbf{a}^{\prime}\right)-B_{1} \oplus \operatorname{diag}\left(\mathbf{a}^{\prime}\right) \in \mathcal{H}_{n}(p, q)$.

For $4 \leq p+q \leq n$, we have $p, q \geq 1$. Therefore, $2 \leq(p-1)+(q-1) \leq n-2$ and $p-1=q-1$ or $p-1=(q-1)+1$. Let $A_{1}=\operatorname{diag}\left(a_{u}, a_{v}\right)$ and $B_{1}=\operatorname{diag}\left(a_{v}, a_{u}\right)$; we have $A_{1}-B_{2} \in \operatorname{In}(1,1)$. Remove $a_{r}, a_{s}$ from a to get $\mathbf{a}^{\prime}$. Since $n \geq 5$, there are at least three distinct entries in $\mathbf{a}^{\prime}$ and each has multiplicity at most $(n-2) / 2$. By the induction assumption, there are $A_{2}, B_{2}$ both with vector of eigenvalues $\mathbf{a}^{\prime}$ such that $A_{2}-B_{2} \in \mathcal{H}_{n-2}(p-1, q-1)$. Thus, $A_{1} \oplus A_{2}-B_{1} \oplus B_{2} \in \mathcal{H}_{n}(p, q)$.

(3) Suppose conditions (i) and (ii) do not hold. Using the notation in Theorem 3.3. we see that $(p, q) \in \operatorname{In}(\mathbf{a}, \mathbf{b})$ for

$$
(p, q) \in\left\{\left(p_{j}, q_{0}\right): 0 \leq j \leq k\right\} \cup\left\{\left(p_{k}, q_{j}\right): 1 \leq j \leq Q_{k}\right\} .
$$

Thus, we have the desired rank values.

By Theorem 2.1, we can construct matrices $A$ and $B$ with the asserted block structure.

It is clear that $X, Y \in \mathcal{H}_{n}$ have the same eigenvalues if and only if $X-\mu I$ and $Y-\mu I$ have the same inertia (or rank) for all eigenvalues $\mu$ of $Y$. Thus, we can describe the eigenvalues of $A-B$ in terms of the inertia of $A-B-\mu I$ for different real numbers $\mu$. In particular, we have the following necessary condition for $c_{1} \geq \cdots \geq c_{n}$ to be the eigenvalues of $A-B$ with $(A, B) \in \mathcal{H}_{n}(\mathbf{a}) \times \mathcal{H}_{n}(\mathbf{b})$.

Proposition 5.2 Let $\mathbf{a}=\left(a_{1}, \ldots, a_{n}\right), \mathbf{b}=\left(b_{1}, \ldots, b_{n}\right)$, and $\mathbf{c}=\left(c_{1}, \ldots, c_{n}\right)$ be real vectors with entries arranged in descending order. Suppose $\mathrm{c}$ has distinct entries $c_{1}>\cdots>c_{t}$ with multiplicities $m_{1}, \ldots, m_{t}$, respectively, and suppose there exists $(A, B) \in \mathcal{H}_{n}(\mathbf{a}) \times \mathcal{H}_{n}(\mathbf{b})$ such that $A-B \in \mathcal{H}_{n}(\mathbf{c})$. Set $u_{0}=0, u_{j}=m_{1}+\cdots+m_{j-1}$ for $j \in\{1, \ldots, t\}, v_{j}=m_{j+1}+\cdots+m_{t}$ for $j \in\{1, \ldots, t-1\}$ and $v_{t}=0$. Then for $j \in\{1, \ldots, t\}$,

(i) $\left(a_{1}-c_{j}, \ldots, a_{n-v_{j}}-c_{j}\right)-\left(b_{v_{j}+1}, \ldots, b_{n}\right)$ is nonnegative with at least $u_{j}$ positive entries.

(ii) $\left(b_{1}, \ldots, b_{n-u_{j}}\right)-\left(a_{u_{j}+1}-c_{j}, \ldots, a_{n}-c_{j}\right)$ is nonnegative with at least $v_{j}$ positive entries. 
Remark 5.3 Let $\mathbf{a}=\left(a_{1}, \ldots, a_{n}\right)$ and $\mathbf{b}=\left(b_{1}, \ldots, b_{n}\right)$ with entries arranged in descending order. Then there exist $A, B \in \mathcal{H}_{n}$ with vector of eigenvalues a and $\mathbf{b}$ such that $A-B$ has an eigenvalue $\mu$ with multiplicity $t$ if and only if there is a matrix of the form $\tilde{A}-B$ with rank $n-t$, where $\tilde{A}+\mu I \in \mathcal{H}_{n}(\mathbf{a})$ and $B \in \mathcal{H}_{n}(\mathbf{b})$. Hence, we can use Theorem 5.1 to determine whether there is $(A, B) \in \mathcal{H}_{n}(\mathbf{a}) \times \mathcal{H}_{n}(\mathbf{b})$ such that $A-B$ has an eigenvalue $\mu$ with multiplicity $t$. In Corollaries 5.6 and 5.7, we will apply Theorem 2.1 to give a more precise location of the multiple eigenvalue $\mu$. As a byproduct, we determine the function $f(\mu)$ defined as the minimum rank of a matrix of the form $A-B-\mu I$ with $(A, B) \in \mathcal{H}_{n}(\mathbf{a}) \times \mathcal{H}_{n}(\mathbf{b})$ for given real vectors a and $\mathbf{b}$.

The following notation will be used for the rest of this section.

Notation 5.4 Let $\mathbf{a}=\left(a_{1}, \ldots, a_{n}\right), \mathbf{b}=\left(b_{1}, \ldots, b_{n}\right)$ be real vectors with entries arranged in descending order. For $0 \leq t \leq n-1$, let

$\alpha_{t}=\max \left\{a_{j+t}-b_{j}: 1 \leq j \leq n-t\right\} \quad$ and $\quad \beta_{t}=\min \left\{a_{j}-b_{j+t}: 1 \leq j \leq n-t\right\}$.

For $\mu \in \mathbf{R}$, let $p_{0}(\mu)$ and $q_{0}(\mu)$ be defined as in (3.1), (3.2) with $a_{j}$ replaced by $a_{j}-\mu$.

Note that $p_{0}(\mu)+q_{0}(\mu)$ will be the minimum rank of a matrix of the form $A-B-\mu I$ with $(A, B) \in \mathcal{H}_{n}(\mathbf{a}) \times \mathcal{H}_{n}(\mathbf{b})$.

Proposition 5.5 Let $\mathbf{a}$ and $\mathbf{b}$ be real vectors with entries arranged in descending order. We have

$$
\alpha_{n-1} \leq \alpha_{n-2} \leq \cdots \leq \alpha_{0} \quad \text { and } \quad \beta_{0} \leq \beta_{1} \leq \cdots \leq \beta_{n-1} .
$$

Moreover, the following conditions hold for the functions $p_{0}(\mu), q_{0}(\mu)$, and $p_{0}(\mu)+$ $q_{0}(\mu)$.

(i) $p_{0}(\mu)$ is a decreasing step function in $\mu \in \mathbf{R}$ such that $p_{0}(\mu)=n$ for $\mu<\alpha_{n-1}$, $p_{0}(\mu)=0$ for $\mu \geq \alpha_{0}$, and $p_{0}(\mu)=t$ if $\mu$ in the interval $\left[\alpha_{t}, \alpha_{t-1}\right)$ for $1 \leq t \leq$ $n-1$;

(ii) $q_{0}(\mu)$ is an increasing step function in $\mu \in \mathbf{R}$ such that $q_{0}(\mu)=0$ for $\mu \leq \beta_{0}$, $q_{0}(\mu)=n$ for $\mu>\beta_{n-1}$, and $q_{0}(\mu)=t$ if $\mu$ is in the interval $\left(\beta_{t-1}, \beta_{t}\right]$ for $1 \leq t \leq n-1$.

(iii) If $\alpha_{s}=\beta_{t}$ for some $0 \leq s, t \leq n-1$, then there exists $\delta>0$ such that $p_{0}(\mu)+q_{0}(\mu)>p_{0}\left(\alpha_{s}\right)+q_{0}\left(\alpha_{s}\right)$ for all $0<\left|\mu-\alpha_{s}\right|<\delta$.

(iv) If $\mu \neq \alpha_{t}, \beta_{t}$ for all $0 \leq t \leq n-1$, then $p_{0}(\cdot)+q_{0}(\cdot)$ is locally constant at $\mu$.

Proof For $1 \leq t \leq n-1$ and $1 \leq j \leq n-t$ we have $a_{j+t}-b_{j} \leq a_{j+(t-1)}-b_{j}$. Therefore, $\alpha_{t} \leq \alpha_{t-1}$. Similarly, $\beta_{t} \geq \beta_{t-1}$.

By (3.1) and (3.2), we have

$$
\begin{aligned}
& p_{0}(\mu)= \begin{cases}n & \text { if } \mu<a_{n}-b_{1}, \\
\min \left\{t: \mu \geq \alpha_{t}\right\} & \text { otherwise, }\end{cases} \\
& q_{0}(\mu)= \begin{cases}n & \text { if } a_{1}-b_{n}<\mu, \\
\min \left\{t: \mu \leq \beta_{t}\right\} & \text { otherwise, }\end{cases}
\end{aligned}
$$


which implies (i) and (ii).

For (iii), suppose $\alpha_{s}=\beta_{t}$ for some $0 \leq s, t \leq n-1$. By taking $\alpha_{n}=\alpha_{n-1}-1$, $\alpha_{-1}=\alpha_{0}+1, \beta_{-1}=\beta_{0}-1$, and $\beta_{n}=\beta_{n-1}+1$, we may assume that

$$
\begin{aligned}
& \alpha_{s+1}<\alpha_{s}=\alpha_{s-1}=\cdots=\alpha_{s^{\prime}}<\alpha_{s^{\prime}-1}, \\
& \beta_{t-1}<\beta_{t}=\beta_{t+1}=\cdots=\beta_{t^{\prime}}<\beta_{t^{\prime}+1} .
\end{aligned}
$$

Let $\delta=\min \left\{\alpha_{s}-\alpha_{s+1}, \alpha_{s^{\prime}-1}-\alpha_{s^{\prime}}, \beta_{t}-\beta_{t-1}, \beta_{t^{\prime}+1}-\beta_{t^{\prime}}\right\}>0$. We have

$$
\begin{aligned}
p_{0}(\mu)+q_{0}(\mu) & = \begin{cases}s+t+1 & \text { if } 0<\alpha_{s}-\mu<\delta, \\
s^{\prime}+t^{\prime}+1 & \text { if } 0<\mu-\alpha_{s}<\delta\end{cases} \\
& >s^{\prime}+t=p_{0}\left(\alpha_{s^{\prime}}\right)+q_{0}\left(\beta_{t}\right)=p_{0}\left(\alpha_{s}\right)+q_{0}\left(\alpha_{s}\right)
\end{aligned}
$$

(iv) follows from (i) and (ii).

Note that the function $g(\mu)$ defined as the maximum rank of a matrix of the form $A-B-\mu I$ with $(A, B) \in \mathcal{H}_{n}(\mathbf{a}) \times \mathcal{H}_{n}(\mathbf{b})$ is easy to determine, namely, it is equal to $g(\mu)=\min \{n, 2 n-m(\mu)\}$ with $m(\mu)$ equal to the maximum multiplicity of an entry in the vector $\left(a_{1}-\mu, \ldots, a_{n}-\mu, b_{1}, \ldots, b_{n}\right)$.

Similarly, one can consider $P_{\ell}(\mu)$ and $Q_{k}(\mu)$ defined as the maximum number of positive and negative eigenvalues of a matrix of the form $A-B-\mu I$ with $(A, B) \in$ $\mathcal{H}_{n}(\mathbf{a}) \times \mathcal{H}(\mathbf{b})$. We omit their discussion.

The following corollary concerns the possible multiplicities for $\mu \in \mathbf{R}$ to be an eigenvalue of $A-B$ with $(A, B) \in \mathcal{H}_{n}(\mathbf{a}) \times \mathcal{H}_{n}(\mathbf{b})$.

Corollary 5.6 Let $\mathbf{a}$ and $\mathbf{b}$ be real vectors with entries arranged in descending order. Suppose $a_{n}-b_{1} \leq \mu \leq a_{1}-b_{n}$. Then there exist $s, t \in\{0, \ldots, n-1\}$ such that $\mu \in\left[\alpha_{s}, \alpha_{s-1}\right) \cap\left(\beta_{t-1}, \beta_{t}\right]$, where we take $\alpha_{-1}>\beta_{n-1}$ and $\beta_{-1}<\alpha_{n-1}$.

(i) Suppose $(A, B) \in \mathcal{H}_{n}(\mathbf{a}) \times \mathcal{H}_{n}(\mathbf{b})$ and $\mu$ is an eigenvalue of $A-B$. Then the multiplicity of $\mu$ is at most $n-s-t$. Furthermore, $A-B$ has at least seigenvalue greater than $\mu$ and at least $t$ eigenvalues less than $\mu$.

(ii) There exists $(A, B) \in \mathcal{H}_{n}(\mathbf{a}) \times \mathcal{H}_{n}(\mathbf{b})$ such that $A-B$ has an eigenvalue $\mu$ with multiplicity $n-s-t$, s eigenvalues greater than $\mu$, and t eigenvalues less than $\mu$.

To facilitate the comparison of our results and those in the literature, we present the next corollary in terms of $A+B$ with $(A, B) \in \mathcal{H}_{n}(\mathbf{a}) \times \mathcal{H}_{n}(\mathbf{b})$. We use the following notation. Let $\mathbf{a}=\left(a_{1}, \ldots, a_{n}\right), \mathbf{b}=\left(b_{1}, \ldots, b_{n}\right)$, and $\mathbf{c}=\left(c_{1}, \ldots, c_{n}\right)$ with entries arranged in descending order. For each $1 \leq k \leq n$, let

$$
L_{k}=\max \left\{a_{i}+b_{j}: i+j=n+k\right\} \quad \text { and } \quad R_{k}=\min \left\{a_{i}+b_{j}: i+j=k+1\right\} .
$$

Suppose $A, B \in \mathcal{H}_{n}$ and $C=A+B$ have eigenvalues $\mathbf{a}, \mathbf{b}$, and $\mathbf{c}$. Then it follows from Weyl's inequalities [13] that $L_{k} \leq c_{k} \leq R_{k}$. Conversely, for every $1 \leq k \leq n$ and $c \in\left[L_{k}, R_{k}\right]$, there exist $A, B \in \mathcal{H}_{n}$, and $C=A+B$ with eigenvalues $\mathbf{a}$, $\mathbf{b}$, and c such that $c_{k}=c$. However, for two distinct $1 \leq k<k^{\prime} \leq n$ and $c \in\left[L_{k}, R_{k}\right]$, $c^{\prime} \in\left[L_{k^{\prime}}, R_{k^{\prime}}\right]$, there may not exist $A, B \in \mathcal{H}_{n}$ and $C=A+B$ with eigenvalues $\mathbf{a}, \mathbf{b}$, 
and $\mathrm{c}$ such that $c_{k}=c$ and $c_{k^{\prime}}=c^{\prime}$; see the example in [7, p. 215]. Nevertheless, by replacing $b_{j}$ with $-b_{n+1-j}$ and putting $s=k-1$ and $t=n-k^{\prime}$, the second part of Corollary 5.6 can be rephrased in the following form.

Corollary 5.7 Let $\mathbf{a}=\left(a_{1}, \ldots, a_{n}\right)$ and $\mathbf{b}=\left(b_{1}, \ldots, b_{n}\right)$ with entries arranged in descending order and $\mu \in\left[L_{k}, L_{k-1}\right) \cap\left(R_{k^{\prime}+1}, R_{k^{\prime}}\right]$. Then there exists $(A, B) \in$ $\mathcal{H}_{n}(\mathbf{a}, \mathbf{b})$ such that $C=A+B$ has a vector of eigenvalues $\mathbf{c}$ with

$$
c_{k-1}<\mu=c_{k}=c_{k+1}=\cdots=c_{k^{\prime}}<c_{k^{\prime}+1} .
$$

We remark that Corollary 5.7 can also be deduced from the results in [1].

\section{Additional Results and Remarks}

Proposition 6.1 Let $\mathbf{a}, \mathbf{b}$ be given. There are $1 \times n$ vectors $\mathbf{a}^{\prime}$ and $\mathbf{b}^{\prime}$ with integral entries arranged in descending order such that $\operatorname{In}(\mathbf{a}, \mathbf{b})=\operatorname{In}\left(\mathbf{a}^{\prime}, \mathbf{b}^{\prime}\right)$. Moreover, for each $(p, q) \in \operatorname{In}(\mathbf{a}, \mathbf{b})$ there is $A \in \mathcal{H}_{n}\left(\mathbf{a}^{\prime}\right)$ and $B \in \mathcal{H}_{n}\left(\mathbf{b}^{\prime}\right)$ such that $A-B \in \operatorname{In}\left(\mathbf{a}^{\prime}, \mathbf{b}^{\prime}\right)$ has integer eigenvalues.

Proof We can construct $\mathbf{a}^{\prime}$ and $\mathbf{b}^{\prime}$ as follows. Use the entries of $\mathbf{a}$ and $\mathbf{b}$ to form a vector $\gamma=\left(\gamma_{1}, \ldots, \gamma_{2 n}\right)$ with entries in descending order. We always put the entries of a first if an entry appears in both vectors. Suppose $\gamma$ has $m$ distinct entries $\mu_{1}>\cdots>\mu_{m}$. Then replace the entries $\mu_{i}$ in $\mathbf{a}$ and $\mathbf{b}$ by the integer $i$ for each $i \in\{1, \ldots, m\}$ to get the vectors $\mathbf{a}^{\prime}$ and $\mathbf{b}^{\prime}$. By Theorem 2.1 and the construction of $\mathbf{a}^{\prime}$ and $\mathbf{b}^{\prime}$, we see that $(p, q) \in \operatorname{In}(\mathbf{a}, \mathbf{b})$ if and only if $(p, q) \in \operatorname{In}\left(\mathbf{a}^{\prime}, \mathbf{b}^{\prime}\right)$. Moreover, by Theorem 2.1, for each $(p, q) \in \operatorname{In}\left(\mathbf{a}^{\prime}, \mathbf{b}^{\prime}\right)$ we can construct

$$
A=A_{1} \oplus \cdots \oplus A_{p+q} \in \mathcal{H}_{n}\left(\mathbf{a}^{\prime}\right) \quad \text { and } \quad B=B_{1} \oplus \cdots \oplus B_{p+q} \in \mathcal{H}_{n}\left(\mathbf{b}^{\prime}\right)
$$

such that $A_{i}-B_{i}$ is a rank one positive semi-definite for $i=1, \ldots, p$, and $A_{i}-B_{i}$ is a rank one negative semi-definite for $i=p+1, \ldots, p+q$. Since $A_{i}$ and $B_{i}$ have integral eigenvalues, the only nonzero eigenvalue of $A_{i}-B_{i}$, which equals $\operatorname{tr}\left(A_{i}-B_{i}\right)$, is again an integer. So, the last assertion holds.

Suppose a, b, c have nonnegative integral entries. It is known that there exist $(A, B) \in \mathcal{H}_{n}(\mathbf{a}) \times \mathcal{H}_{n}(\mathbf{b})$ such that $A-B \in \mathcal{H}_{n}(\mathbf{c})$ if and only if one can obtain the Young diagram associated with $\left(a_{1}, \ldots, a_{n}\right)$ from the Young diagrams associated with $\left(b_{1}, \ldots, b_{n}\right)$ and $\left(c_{1}, \ldots, c_{n}\right)$ according to the Little-Richardson rules; see [7]. Thus, we have the following result.

Proposition 6.2 Let $\mathbf{a}=\left(a_{1}, \ldots, a_{n}\right)$ and $\mathbf{b}=\left(b_{1}, \ldots, b_{n}\right)$ have positive integral entries arranged in descending order. Then there is a vector $\mathbf{c}=\left(c_{1}, \ldots, c_{n}\right)$ with positive integral entries arranged in descending order and $c_{p+1}=\cdots=c_{n-q+1}=\mu$ for a given integer $\mu$ such that one can obtain the Young diagram associated with a from the Young diagrams associated with $\mathbf{b}$ and $\mathbf{c}$ according to the Little-Richardson rules if and only if

$$
\left(a_{1}-\mu, \ldots, a_{n-q}-\mu\right) \geq_{p}\left(b_{q+1}, \ldots, b_{n}\right)
$$

and

$$
\left(b_{1}, \ldots, b_{n-p}\right) \geq_{q}\left(a_{p+1}-\mu, \ldots, a_{n}-\mu\right) .
$$


In connection with our discussion, it would be interesting to solve the following.

Problem 3 Deduce and extend Proposition 6.2 using the theory of algebraic combinatorics. In particular, for given real vectors $\mathbf{a}$ and $\mathbf{b}$ with integral entries, determine the conditions for the existence of an integral vector $\mathbf{c}$ with certain prescribed entries such that the Young diagram corresponding to a can be obtained from those of $\mathbf{b}$ and c according to the Littlewood-Richardson rules.

Problem 4 Extend our results to the sum of $k$ Hermitian matrices for $k>2$. In other words, determine all possible inertia values and ranks of matrices in $\mathcal{H}_{n}\left(\mathbf{a}_{1}\right)+\cdots+\mathcal{H}_{n}\left(\mathbf{a}_{k}\right)$ for given $1 \times n$ real vectors $\mathbf{a}_{1}, \ldots, \mathbf{a}_{k}$ with entries arranged in descending order.

Note that the problem of finding the relation between the eigenvalues of $A_{1}$, $\ldots, A_{k}$ and those of $A_{0}=A_{1}+\cdots+A_{k}$ can be reformulated as the problem of finding the necessary and sufficient conditions for the existence of Hermitian matrices $A_{0}, A_{1}, \ldots, A_{k}$ with prescribed eigenvalues such that $A_{0}-\sum_{j=1}^{k} A_{j}$ has rank 0 . Thus, it can be viewed as a special case of Problem 4 To determine whether there are $A_{1}, \ldots, A_{k} \in \mathcal{H}_{n}$ with prescribed eigenvalues such that $A_{1}+\cdots+A_{k}$ has rank one, one may reduce the problem to the study of the existence of $A_{1}, \ldots, A_{k} \in \mathcal{H}_{n}$ with prescribed eigenvalues such that $A_{1}+\cdots+A_{k}$ has eigenvalue $\mu, 0, \ldots, 0$ with $\mu=\operatorname{tr}\left(A_{1}+\cdots+A_{k}\right)$. Then the results in [7] can be used to solve the problem. In general, it seems difficult to determine if there exist $A_{1}, \ldots, A_{k}$ with prescribed eigenvalues such that $A_{1}+\cdots+A_{k}$ has rank $r$ with $r \in\{2, \ldots, n\}$.

Note added in proof We thank Professor Wing Suet Li for some helpful dicussions about the connection between the interesting preprint [1] and our work. In [1, Proposition 5.1], the authors determined the conditions on $1 \times n$ vectors $\mathbf{a}_{0}, \mathbf{a}_{1}, \ldots, \mathbf{a}_{k}$ with some of the their entries specified so that one can fill in the missing entries to get vectors $\tilde{\mathbf{a}}_{0}, \ldots, \tilde{\mathbf{a}}_{k}$ with entries arranged in descending order and Hermitian matrices $A_{j} \in \mathcal{H}_{n}\left(\tilde{\mathbf{a}}_{j}\right)$ for $j=0,1, \ldots, k$ satisfying $A_{0}=A_{1}+\cdots+A_{k}$. Evidently, there exists $A_{0} \in \mathcal{H}\left(\mathbf{a}_{1}\right)+\cdots+\mathcal{H}\left(\mathbf{a}_{k}\right)$ with inertia $(p, q, n-p-q)$ for given $1 \times n$ real vectors $\mathbf{a}_{1}, \ldots, \mathbf{a}_{k}$ if and only if there exist $\delta, \varepsilon>0$ and $A_{0} \in \mathcal{H}\left(\mathbf{a}_{1}\right)+\cdots+\mathcal{H}\left(\mathbf{a}_{k}\right)$ with eigenvalues $\mu_{1} \geq \cdots \geq \mu_{n}$ such that $\left(\mu_{p}, \ldots, \mu_{n-q+1}\right)=(\delta, 0, \ldots, 0,-\varepsilon)$. Using the result in [1], one can determine whether the positive numbers $\delta, \varepsilon$ exist by checking whether a polytope defined a large number of inequalities in terms of entries of $\mathbf{a}_{1}, \ldots, \mathbf{a}_{k}$ has non-empty interior; see also Buch [2]. For $k=2$, our Theorem 2.1 shows that the very involved conditions can be reduced to (i) and (ii).

\section{References}

[1] H. Bercovici, W. S. Li and D. Timotin, The Horn conjecture for sums of compact selfadjoint operators. http://arXiv.org/abs/0709.1088.

[2] A. S. Buch, Eigenvalues of Hermitian matrices with positive sum of bounded rank. Linear Algebra Appl. 418(2006), no. 2-3, 480-488. doi:10.1016/j.laa.2006.02.024

[3] M. D. Choi and P. Y. Wu, Convex combinations of projections. Linear Algebra Appl. 136(1990), 25-42. doi:10.1016/0024-3795(90)90019-9 
[4] Finite-rank perturbations of positive operators and isometries. Studia Math. 173(2006), no. 1, 73-79. doi:10.4064/sm173-1-5

[5] J. Day, W. So, and R. C. Thompson, The spectrum of a Hermitian matrix sum. Linear Algebra Appl. 280(1998), no. 2-3, 289-332. doi:10.1016/S0024-3795(98)10019-8

[6] K. Fan and G. Pall, Imbedding conditions for Hermitian and normal matrices. Canad. J. Math. 9(1957), 298-304.

[7] W. Fulton, Eigenvalues, invariant factors, highest weights, and Schubert calculus. Bull. Amer. Math. Soc. 37(2000), no. 3, 209-249. doi:10.1090/S0273-0979-00-00865-X

[8] A. Horn, Eigenvalues of sums of Hermitian matrices. Pacific J. Math 12(1962), 225-241.

[9] A. A. Klyachko, Stable bundles, representation theory and Hermitian operators. Selecta Math. 4(1998), no. 2, 419-445. doi:10.1007/s000290050037

[10] A. Knutson and T. Tao, The honeycomb model of $\mathrm{GL}_{n}(c)$ tensor products. I. Proof of the saturation conjecture. J. Amer. Math. Soc. 12(1999), no. 4, 1055-1090. doi:10.1090/S0894-0347-99-00299-4

[11] R. C. Thompson and L. J. Freede, On the eigenvalues of sums of Hermitian matrices. Linear Algebra and Appl. 4(1971), 369-376. doi:10.1016/0024-3795(71)90007-3

[12] On the eigenvalues of sums of Hermitian matrices. II. Aequationes Math 5(1970), $103-115$. doi:10.1007/BF01819276

[13] H. Weyl, Das asymtotische Verteilungsgesetz der Eigenwerte lineare partieller Differentialgleichungen. Math. Ann. 71(1912), no. 4, 441-479 doi:10.1007/BF01456804

(Li) Department of Mathematics, College of William and Mary, Williamsburg, VA 23185, USA

and

University of Hong Kong

e-mail: ckli@math.wm.edu

(Poon) Department of Mathematics, Iowa State University, Ames, IA 50011, USA

e-mail: ytpoon@iastate.edu 